TRANSACTIONS OF THE

AMERICAN MATHEMATICAL SOCIETY

Volume 351, Number 5, Pages 2025-2054

$\mathrm{S} 0002-9947(99) 02142-\mathrm{X}$

Article electronically published on January 27, 1999

\title{
HARNACK INEQUALITY FOR THE LINEARIZED PARABOLIC MONGE-AMPÈRE EQUATION
}

\author{
QINGBO HUANG
}

\begin{abstract}
In this paper we prove the Harnack inequality for nonnegative solutions of the linearized parabolic Monge-Ampère equation

$$
u_{t}-\operatorname{tr}\left(\left(D^{2} \phi(x)\right)^{-1} D^{2} u\right)=0
$$

on parabolic sections associated with $\phi(x)$, under the assumption that the Monge-Ampère measure generated by $\phi$ satisfies the doubling condition on sections and the uniform continuity condition with respect to Lebesgue measure. The theory established is invariant under the group $A T(n) \times A T(1)$, where $A T(n)$ denotes the group of all invertible affine transformations on $\mathbf{R}^{n}$.
\end{abstract}

\section{INTRODUCTION}

In this paper we consider the Harnack inequality for nonnegative solutions of the following equation:

$$
L_{\phi} u=u_{t}-\operatorname{tr}\left(\left(D^{2} \phi(x)\right)^{-1} D^{2} u\right)=0,
$$

where $u_{t}=\frac{\partial u}{\partial t}, D^{2} u$ denotes the Hessian of $u$ in the $x$ variable, $\left(D^{2} \phi(x)\right)^{-1}$ is the inverse matrix of the Hessian of a strictly convex smooth function $\phi(x)$ defined in $\mathbf{R}^{n}$ (i.e., $D^{2} \phi>0$ ), and $\operatorname{tr}(A)$ means the trace of the matrix $A$.

There is a family of convex sets called sections associated with $\phi$. For $x \in \mathbf{R}^{n}$, $r>0$, we define the section $S(x, r)$ by

$$
S(x, r)=S_{\phi}(x, r)=\left\{y \in \mathbf{R}^{n}: \phi(y)<\ell_{x}(y)+r\right\},
$$

where $\ell_{x}$ denotes the supporting hyperplane of $\phi$ at $x$. Sections play an important role in the investigation of Monge-Ampère equation and the linearized MongeAmpère equation, see [Ca1], [Ca2], [Ca-Gu1], and [Ca-Gu2]. We will also consider parabolic sections $Q(z, r)$ associated with $\phi$ defined by

$$
Q(z, r)=Q_{\phi}(z, r)=S(x, r) \times(t-r, t],
$$

where $z=(x, t)$ and $r>0$.

Recently, in [Ca-Gu2] a Harnack inequality has been established in terms of sections for nonnegative solutions of the linearized Monge-Ampère equation

$$
\operatorname{tr}\left(\left(D^{2} \phi\right)^{-1} D^{2} u\right)=0 .
$$

The theory developed by these authors is invariant under invertible affine transformations in $\mathbf{R}^{n}$.

Received by the editors December 15, 1996 and, in revised form, May 13, 1997.

1991 Mathematics Subject Classification. Primary 35K10; Secondary 35B45.

Key words and phrases. Harnack inequality, affine invariant, linear parabolic Monge-Ampère equation, section.

(c)1999 American Mathematical Society 
The main purpose of this paper is to establish Harnack inequality for nonnegative solutions of (1-1) on parabolic sections and thus generalize the result in [Ca-Gu2] from the elliptic to the parabolic case. In case $\phi(x)=\frac{1}{2}|x|^{2},(1-1)$ is the heat equation and all associated parabolic sections are the standard parabolic cylinders. The Harnack inequality for the heat equation is well known; see [M]. In case $\phi(x)=\langle M x, x\rangle$ where $M$ is a positive definite symmetric matrix, we can write $M=M_{1}^{2}$ where $M_{1}$ is positive definite symmetric and the corresponding section $S\left(x_{0}, r\right)$ is of form

$$
S\left(x_{0}, r\right)=\left\{x:\left|M_{1} x-M_{1} x_{0}\right|^{2}<r\right\} .
$$

If $T$ is the affine transformation defined by $T(x, t)=\left(M_{1} x, t\right)$, and $u$ is a solution of (1-1), then $v(y, t)=u\left(T^{-1}(y, t)\right)$ satisfies the heat equation. Therefore, if $u \geq 0$, then by the Harnack inequality for the heat equation it follows that $u$ satisfies Harnack inequality on the parabolic sections, i.e.,

$$
\sup _{Q^{-}} u \leq C \inf _{Q^{+}} u,
$$

where $Q^{+}=S\left(x_{0}, r\right) \times\left(t_{0}-r, t_{0}\right]$ and $Q^{-}=S\left(x_{0}, r\right) \times\left(t_{0}-3 r, t_{0}-2 r\right]$. We will show that a similar result holds for general $\phi$.

Now we state the assumptions and the main result of this paper.

Let $\phi(x)$ be a strictly convex smooth funtion in $\mathbf{R}^{n}$ (i.e., $D^{2} \phi>0$ ) and $\mu$ be the Monge-Ampère measure generated by $\phi$, i.e., $d \mu=\operatorname{det} D^{2} \phi d x$. Throughout this paper, we assume that $\mu$ satisfies the following doubling property in terms of sections:

$$
\mu(S(x, r)) \leq A \mu\left(\frac{1}{2} S(x, r)\right), \quad \text { for } x \in \mathbf{R}^{n}, r>0,
$$

where $A>0$ and $\frac{1}{2} S(x, r)$ denotes $\frac{1}{2}$-dilation of $S(x, r)$ with respect to its center of mass. We also assume that $\mu$ satisfies the uniform continuity condition $\mu_{\infty}$.

Condition $\mu_{\infty}$. For $\delta_{1} \in(0,1)$, there exists $\delta_{2} \in(0,1)$ such that, for any section $S$ and any measurable set $E \subset S$,

$$
\frac{|E|}{|S|}<\delta_{2} \quad \text { implies } \quad \frac{\mu(E)}{\mu(S)}<\delta_{1}
$$

where $|E|$ denotes Lebesgue measure of $E$.

Some examples of measures satisfying (1-2) and (1-3) are those measures whose densities are positive polynomials in $\mathbf{R}^{n}$. Also note that the condition $\mu_{\infty}$ can be viewed as an $A_{\infty}$-weight condition in terms of sections.

By [Ca-Gu2], the condition $\mu_{\infty}$ implies that $\mu$ is a doubling measure in the following sense: there exist $C$ and $0<\alpha<1$ such that

$$
\mu(S(x, r)) \leq C \mu(\alpha S(x, r)),
$$

where $\alpha S$ means $\alpha$-dilation of $S$ with respect to its center of mass.

We also consider the parabolic Monge-Ampère measure $\mathcal{M}$ generated by $\phi(x)-t$, i.e., $d \mathcal{M}=\operatorname{det} D^{2} \phi d x d t=d \mu d t$.

The following theorem is the main result of this paper.

Theorem 1.1. Suppose that $\phi$ satisfies (1-2) and (1-3). Let $u$ be a nonnegative classical solution of (1-1) in $S\left(x_{0}, \bar{\theta} R\right) \times\left(t_{0}-\frac{3}{2} R, t_{0}+2 R\right]$, where $\bar{\theta}$ is a large 
geometric constant. Then

$$
\sup _{Q^{-}} u \leq C \inf _{Q^{+}} u,
$$

where $Q^{+}=S\left(x_{0}, R\right) \times\left(t_{0}+R, t_{0}+2 R\right]$ and $Q^{-}=S\left(x_{0}, R\right) \times\left(t_{0}-R, t_{0}\right]$, and $C$ depends only on $n$, and the structure conditions (1-2) and (1-3).

We mention here that the assumption (1-2) in Theorem 1.1 can be replaced by (1-4) and the conclusion (1-5) is still valid. Indeed, the properties of sections and measures used in the proof of Theorem 1.1 are still valid under the assumptions (1-3) and (1-4). Recalling that (1-3) implies (1-4), we conclude that (1-5) holds if $\phi$ satisfies (1-3).

Also, the theory established here is invariant under the group of affine transformations $A T(n) \times A T(1)$, which is the subgroup of $A T(n+1)$ denoting the group of all invertible affine transformations on $\mathbf{R}^{n+1}$. Let $T_{n} \in A T(n)$ and $T_{1} \in A T(1)$. Define $T_{n+1}$ by

$$
(y, s)=T_{n+1}(x, t)=\left(T_{n} x, T_{1} t\right)=\left(T_{n} x, a t+b\right) .
$$

Let $\bar{u}(y, s)=u\left(T_{n+1}^{-1}(y, s)\right)$ and $\bar{\phi}(y)=a \phi\left(T_{n}^{-1} y\right)$. It is easy to check that if $L_{\phi} u=0$, then

$$
L_{\bar{\phi}} \bar{u}=\frac{\partial \bar{u}}{\partial s}-\operatorname{tr}\left(\left(D^{2} \bar{\phi}\right)^{-1} D^{2} \bar{u}\right)=0,
$$

and

$$
\begin{aligned}
T_{n}\left(S_{\phi}\left(x_{0}, r\right)\right) & =S_{\bar{\phi}}\left(T_{n} x_{0}, a r\right), \\
T_{n+1}\left(Q_{\phi}\left(\left(x_{0}, t_{0}\right), r\right)\right) & =Q_{\bar{\phi}}\left(T_{n+1}\left(x_{0}, t_{0}\right), a r\right), \\
T_{n}\left(\frac{1}{2} S_{\phi}\left(x_{0}, r\right)\right) & =\frac{1}{2} S_{\bar{\phi}}\left(T_{n} x_{0}, a r\right) .
\end{aligned}
$$

Then noting that (1-2) and (1-3) are invariant under any invertible affine transformation $T_{n}$, we conclude that our theory is invariant under $A T(n) \times A T(1)$.

The method to prove Theorem 1.1 is a generalization of that used in [Ca-Gu2] for the elliptic case. Other than the elliptic case, there exists shift of time in estimates in the parabolic case. We have to overcome the difficulty caused by the shift of time which obstruct us to directly apply the procedure in [Ca-Gu2]. On the other hand, we cannot adopt the method in [W] since our estimates do not depend on uniform parabolicity. By the nature of equation (1-1), we will work with the parabolic sections instead of the standard cylinders. Therefore, we have to show that the family of sections has after appropriate affine transformations similar properties to those of the standard parabolic cylinders. Also, we need to develop a variant of the Calderón-Zygmund decomposition in terms of sections. The outline to prove Theorem 1.1 is as follows. The first step is to show that the level sets of solutions have uniform critical density (Lemma 3.1). The second step comes from the first step, i.e., if solutions are large on a section, then solutions are still large after a short time. The third step is to show Lemma 3.3, i.e., if solutions are large on a section, then after a short time of diffusion solutions will be large on a wider section. From these three fundamental estimates and a variant of the Calderón-Zygmund decomposition, the power decay of the distribution functions of the solutions and Harnack inequality will be derived. 
We next describe the linearized parabolic Monge-Ampère equation. Let $\Phi(x, t)$ be a parabolically convex function on $\mathbf{R}^{n+1}$, i.e., convex in $x$ and nonincreasing in $t$. It is easy to check that

$$
\begin{aligned}
& -(\Phi+\lambda u)_{t} \operatorname{det} D^{2}(\Phi+\lambda u) \\
=- & \Phi_{t} \operatorname{det} D^{2} \Phi-\lambda\left(u_{t} \operatorname{det} D^{2} \Phi+\Phi_{t} \operatorname{det} D^{2} \Phi \operatorname{tr}\left(\left(D^{2} \Phi\right)^{-1} D^{2} u\right)\right) \\
& \quad+\cdots-\lambda^{n+1}\left(u_{t} \operatorname{det} D^{2} u\right) .
\end{aligned}
$$

The coefficient of $\lambda$ in (1-6) is called the linearized parabolic Monge-Ampère operator and is denoted by

$$
L_{\Phi}=u_{t} \operatorname{det} D^{2} \Phi+\Phi_{t} \operatorname{det} D^{2} \Phi \operatorname{tr}\left(\left(D^{2} \Phi\right)^{-1} D^{2} u\right) .
$$

Obviously, $L_{\Phi} u=0$ is equivalent to the following equation:

$$
u_{t}+\Phi_{t} \operatorname{tr}\left(\left(D^{2} \Phi\right)^{-1} D^{2} u\right)=0 .
$$

When $\Phi=\phi(x)-t,(1-8)$ gives (1-1).

The organization of the paper is as follows. In $\S 2$, a Besicovitch's type covering lemma is first proved. Then we use it to show a variant of Calderón-Zygmund decomposition in terms of parabolic sections. Some properties of sections are also collected. In $\S 3$, estimates about distribution function of solutions of (1-1) are given. In $\S 4$, the power decay of the distribution function of solutions and Harnack inequality are showed.

\section{A CALDERÓN-Zygmund DECOMPOSITION}

In this section, we will give some properties of sections and measures $\mu$ and $\mathcal{M}$. A variant of Calderón-Zygmund decomposition for parabolic equations in terms of parabolic sections is developed, which is a necessary tool in establishing power decay of distribution function.

We first mention a lemma of Fritz John's type (see [Ca2]): let $S$ be a bounded convex set in $\mathbf{R}^{n}$ with nonempty interior. Consider all the ellipsoids that contain $S$ and are centered at the center of mass of $S$. Let $E$ be the ellipsoid in this class and of minimizing volume. Then $\alpha_{n} E \subset S \subset E$, where $\alpha_{n} E$ means the $\alpha_{n}$-dilation of $E$ with respect to its center of mass. For example, we may take $\alpha_{n}=1 /(n \sqrt{n})$.

Therefore, given a convex set $S$ in $\mathbf{R}^{n}$, there exists an invertible affine transformation $T$ such that

$$
B\left(0, \alpha_{n}\right) \subset T(S) \subset B(0,1),
$$

where $B(x, r)$ denotes the Euclidean ball centered at $x$ and with radius $r$. The set $T(S)$ is called the normalization of $S$ and $T$ is called an affine transformation that normalizes $S$.

Let $T$ be an affine transformation which normalizes $S_{\phi}(x, r)$. Let $\psi_{\lambda}(y)=$ $\frac{1}{\lambda} \phi\left(T^{-1} y\right)$ with $\lambda>0$. Then

$$
T\left(S_{\phi}(x, r)\right)=S_{\psi_{\lambda}}(T x, r / \lambda) .
$$

In the following, we are concerned with properties of parabolic sections. For $z_{0}=\left(x_{0}, t_{0}\right)$ and $r>0$, we define $\widetilde{Q}\left(z_{0}, r\right)$ by

$$
\widetilde{Q}\left(z_{0}, r\right)=\widetilde{Q}_{\phi}\left(z_{0}, r\right)=S_{\phi}\left(x_{0}, r\right) \times\left(t_{0}-r / 2, t_{0}+r / 2\right) .
$$

Note that $\widetilde{Q}\left(z_{0}, r\right)$ is different from $Q\left(z_{0}, r\right)$ defined in $\S 1$ in the position of $z_{0}$. 
Given $\widetilde{Q}\left(z_{0}, r\right)=S\left(x_{0}, r\right) \times\left(t_{0}-r / 2, t_{0}+r / 2\right)$, there exists an affine transformation $T$ which normalizes $S\left(x_{0}, r\right)$. Let $T_{p}:(x, t) \longrightarrow\left(T x,\left(t-t_{0}\right) / r\right)$ and then

$$
K\left(0, \alpha_{n}\right) \subset T_{p}\left(\widetilde{Q}\left(z_{0}, r\right)\right) \subset K(0,1),
$$

where $K(z, r)=B(x, r) \times\left(t-\frac{r^{2}}{2}, t+\frac{r^{2}}{2}\right)$ is the usual parabolic cylinder. The set $T_{p}\left(\widetilde{Q}\left(z_{0}, r\right)\right)$ will be called the normalization of $\widetilde{Q}\left(z_{0}, r\right)$ and $T_{p}$ an affine transformation that normalizes $\widetilde{Q}\left(z_{0}, r\right)$. For $\lambda>0$, set $\psi_{\lambda}(y)=\frac{1}{\lambda} \phi\left(T^{-1} y\right)$; then

$$
T_{p}\left(\widetilde{Q}_{\phi}(z, r)\right)=S_{\psi_{\lambda}}\left(T x, \frac{r}{\lambda}\right) \times\left(-\frac{1}{2}, \frac{1}{2}\right) .
$$

Now we claim the following properties of parabolic sections.

(A) There exist positive constants $K_{1}, K_{2}, K_{3}, \varepsilon_{1}$ and $\varepsilon_{2}$ with the following property: Given two sections $\widetilde{Q}\left(z_{0}, r_{0}\right), \widetilde{Q}(z, r)$ with $r \leq r_{0}$ and $T_{p}$ an affine transformation that normalizes $\widetilde{Q}\left(z_{0}, r_{0}\right)$, if

$$
\widetilde{Q}\left(z_{0}, r_{0}\right) \cap \widetilde{Q}(z, r) \neq \emptyset,
$$

then there exists $z^{\prime}=\left(x^{\prime}, t^{\prime}\right) \in K\left(0, K_{3}\right)$ such that

$$
\begin{gathered}
B\left(x^{\prime}, K_{2}\left(\frac{r}{r_{0}}\right)^{\varepsilon_{2}}\right) \times\left(t^{\prime}-\frac{r}{2 r_{0}}, t^{\prime}+\frac{r}{2 r_{0}}\right) \subset T_{p}(\widetilde{Q}(z, r)) \\
\subset B\left(x^{\prime}, K_{1}\left(\frac{r}{r_{0}}\right)^{\varepsilon_{1}}\right) \times\left(t^{\prime}-\frac{r}{2 r_{0}}, t^{\prime}+\frac{r}{2 r_{0}}\right),
\end{gathered}
$$

and

$$
T_{p} z=\left(T x, t^{\prime}\right) \in B\left(x^{\prime}, \frac{1}{2} K_{2}\left(\frac{r}{r_{0}}\right)^{\varepsilon_{2}}\right) \times\left\{t^{\prime}\right\} .
$$

(B) There exists $\delta>0$ such that for a section $\widetilde{Q}\left(z_{0}, r\right)$ and $z \notin \widetilde{Q}\left(z_{0}, r\right)$, if $T_{p}$ is an affine transformation that normalizes $\widetilde{Q}\left(z_{0}, r\right)$, then

$$
K\left(T_{p}(z), \varepsilon^{\delta}\right) \cap T_{p}\left(\widetilde{Q}\left(z_{0},(1-\varepsilon) r\right)\right)=\emptyset, \quad \text { for } 0<\varepsilon<1 .
$$

(C) Engulfing Property. There exists a constant $\theta>0$ such that if $\widetilde{Q}\left(z_{0}, r\right)$ is a section and $z \in \widetilde{Q}\left(z_{0}, r\right)$, then $\widetilde{Q}\left(z_{0}, r\right) \subset \widetilde{Q}(z, \theta r)$.

(D) Let $\widetilde{Q}_{\psi}\left(z_{0}, 1\right)$ be a normalized section. There exists $p>0$ such that for $0<r<s<1$ and $z \in \widetilde{Q}_{\psi}\left(z_{0}, r\right)$ then

$$
\widetilde{Q}_{\psi}\left(z,(s-r)^{p}\right) \subset \widetilde{Q}_{\psi}\left(z_{0}, s\right) .
$$

Properties (A)-(D) show that the family of parabolic sections has after normalization similar geometric properties to those of usual cylinders. For similar properties of elliptic sections, see [Ca-Gu1], [Ca-Gu2], [Gu-H]. Since parabolic sections are cylinders with bases of elliptic sections, properties (A)-(D) can be easily showed from properties of elliptic sections in [Ca-Gu1], [Ca-Gu2], [Gu-H] and we omit the proof.

We now prove a Besicovitch's type covering lemma.

Lemma 2.1. Let $O \subset \mathbf{R}^{n+1}$ be a bounded set. Suppose that for $z \in O$ a section $\widetilde{Q}(z, r)$ is given such that $r \leq M$ and $M$ is fixed. Denote by $\mathcal{F}$ this family of parabolic sections. Then there exists a countable subfamily of $\mathcal{F},\left\{\widetilde{Q}\left(z_{k}, r_{k}\right)\right\}_{k=1}^{\infty}$, with the following properties:

(i) $O \subset \bigcup_{k=1}^{\infty} \widetilde{Q}\left(z_{k}, r_{k}\right)$. 
(ii) $z_{k} \notin \bigcup_{j<k} \widetilde{Q}\left(z_{j}, r_{j}\right), \quad \forall k \geq 2$.

(iii) For $\varepsilon>0$ small (smallness depending only on the constants in $(A)$ and $(B)$ ) we have that the family $\mathcal{F}_{\varepsilon}=\left\{\widetilde{Q}\left(z_{k},(1-\varepsilon) r_{k}\right)\right\}_{k=1}^{\infty}$ has bounded overlaps. More precisely

$$
\sum_{k=1}^{\infty} \chi_{\widetilde{Q}\left(z_{k},(1-\varepsilon) r_{k}\right)}(z) \leq C_{0} \log \frac{1}{\varepsilon},
$$

where $C_{0}$ depends only on the constants in $(A)$ and $(B) ; \chi_{E}$ denotes the characteristic function of $E$.

Proof. The proof is essentially similar to that of Lemma 1 in [Ca-Gu1]. We may assume $M=\sup \{r: \widetilde{Q}(z, r) \in \mathcal{F}\}$. Let

$$
\mathcal{F}_{0}=\left\{\widetilde{Q}(z, r): \frac{M}{2}<r \leq M, \widetilde{Q}(z, r) \in \mathcal{F}\right\},
$$

and

$$
O_{0}=\left\{z: \widetilde{Q}(z, r) \in \mathcal{F}_{0}\right\} .
$$

Pick $\widetilde{Q}\left(z_{1}, r_{1}\right) \in \mathcal{F}_{0}$. If $O_{0} \subset \widetilde{Q}\left(z_{1}, r_{1}\right)$, we stop. Otherwise, pick $\widetilde{Q}\left(z_{2}, r_{2}\right) \in \mathcal{F}_{0}$ with $z_{2} \in O_{0} \backslash \widetilde{Q}\left(z_{1}, r_{1}\right)$. If $O_{0} \subset \widetilde{Q}\left(z_{1}, r_{1}\right) \cup \widetilde{Q}\left(z_{2}, r_{2}\right)$, we stop. Otherwise we continue this process. Thus, in this way we construct a subfamily $\mathcal{F}_{0}^{\prime}=\left\{\widetilde{Q}\left(z_{i}^{0}, r_{i}^{0}\right)\right\}_{i=1}^{\infty}$ of $\mathcal{F}_{0}$ with $z_{k}^{0} \in O_{0} \backslash \bigcup_{j<k} \widetilde{Q}\left(z_{j}^{0}, r_{j}^{0}\right)$. Actually, we will show later that $\mathcal{F}_{0}^{\prime}$ has only finite number of members.

Next consider the family $\mathcal{F}_{1}=\left\{\widetilde{Q}(z, r) \in \mathcal{F}: \frac{M}{4}<r \leq \frac{M}{2}\right\}$. Let

$$
O_{1}=\left\{z: \widetilde{Q}(z, r) \in \mathcal{F}_{1} \text { and } z \notin \bigcup_{i=1}^{\infty} \widetilde{Q}\left(z_{i}^{0}, r_{i}^{0}\right)\right\} .
$$

We repeat the construction above for the set $O_{1}$ and obtain a family of sections denoted by

$$
\mathcal{F}_{1}^{\prime}=\left\{\widetilde{Q}\left(z_{i}^{1}, r_{i}^{1}\right)\right\}_{i=1}^{\infty},
$$

with $z_{k}^{1} \in O_{1} \backslash \bigcup_{j<k} \widetilde{Q}\left(z_{i}^{1}, r_{i}^{1}\right)$.

We continue this process and in the $k$ th-stage we consider the family $\mathcal{F}_{k}=$ $\left\{\widetilde{Q}(z, r) \in \mathcal{F}: M 2^{-(k+1)}<r \leq M 2^{-k}\right\}$ and the set

$$
O_{k}=\left\{z: \widetilde{Q}(z, r) \in \mathcal{F}_{k} \text { and } z \notin \bigcup_{l=0}^{k-1} \bigcup_{i=1}^{\infty} \widetilde{Q}\left(z_{i}^{l}, r_{i}^{l}\right)\right\} .
$$

As before, we obtain a family of sections denoted by $\mathcal{F}_{k}^{\prime}=\left\{\widetilde{Q}\left(z_{i}^{k}, r_{i}^{k}\right)\right\}_{i=1}^{\infty}$ with $z_{i}^{k} \in O_{k} \backslash \bigcup_{j<i} \widetilde{Q}\left(z_{j}^{k}, r_{j}^{k}\right)$.

We show below that the collection of all sections in all $\mathcal{F}_{k}^{\prime}$ is the family that satisfies the conclusions of Lemma 2.1.

Let us first show that each generation $\mathcal{F}_{k}^{\prime}$ has bounded overlapping. Suppose that

$$
z \in \widetilde{Q}\left(z_{j_{1}}^{k}, r_{j_{1}}^{k}\right) \cap \cdots \cap \widetilde{Q}\left(z_{j_{N}}^{k}, r_{j_{N}}^{k}\right),
$$

where $\widetilde{Q}\left(z_{j_{i}}^{k}, r_{j_{i}}^{k}\right) \in \mathcal{F}_{k}^{\prime}$. For simplicity, set $z_{j_{i}}^{k}=z_{i}, r_{j_{i}}^{k}=r_{i}$, and let $\widetilde{Q}\left(z_{0}, r_{0}\right)$ be the section in $\left\{\widetilde{Q}\left(z_{i}, r_{i}\right)\right\}_{i=1}^{N}$ with $r_{0}=\max \left\{r_{i}: 1 \leq i \leq N\right\}$. We may assume 
by construction that $z_{l} \notin \widetilde{Q}\left(z_{i}, r_{i}\right)$ for $l>i$. By $(\mathrm{A})$, there exists $z_{i}^{\prime}=\left(x_{i}, t_{i}\right) \in$ $K\left(0, K_{3}\right)$ such that

$$
\begin{gathered}
B\left(x_{i}, K_{2}\left(\frac{r_{i}}{r_{0}}\right)^{\varepsilon_{2}}\right) \times\left(t_{i}-\frac{r_{i}}{2 r_{0}}, t_{i}+\frac{r_{i}}{2 r_{0}}\right) \subset T_{p}\left(\widetilde{Q}\left(z_{i}, r_{i}\right)\right) \\
\subset B\left(x_{i}, K_{1}\left(\frac{r_{i}}{r_{0}}\right)^{\varepsilon_{1}}\right) \times\left(t_{i}-\frac{r_{i}}{2 r_{0}}, t_{i}+\frac{r_{i}}{2 r_{0}}\right),
\end{gathered}
$$

and

$$
T_{p}\left(z_{i}\right) \in B\left(x_{i}, \frac{1}{2} K_{2}\left(\frac{r_{i}}{r_{0}}\right)^{\varepsilon_{2}}\right) \times\left\{t_{i}\right\}
$$

where $1 \leq i \leq N$ and $T_{p}$ is an affine transformation that normalizes $\widetilde{Q}\left(z_{0}, r_{0}\right)$. Since $z_{l} \notin \widetilde{Q}\left(z_{i}, r_{i}\right)$, by $(2-3)$, we have that for $l>i$

$$
T_{p}\left(z_{l}\right) \notin B\left(x_{i}, K_{2}\left(\frac{r_{i}}{r_{0}}\right)^{\varepsilon_{2}}\right) \times\left(t_{i}-\frac{r_{i}}{2 r_{0}}, t_{i}+\frac{r_{i}}{r_{0}}\right) .
$$

This together with (2-4) and $\frac{1}{2} \leq \frac{r_{i}}{r_{0}} \leq 1$ implies that

$$
\left|T_{p}\left(z_{l}\right)-T_{p}\left(z_{i}\right)\right|>C, \quad l>i,
$$

where $C$ depends only $K_{2}$ and $\varepsilon_{2}$. By $(2-3)$, it is easy to see that $T_{p}\left(\widetilde{Q}\left(z_{i}, r_{i}\right)\right) \subset Q$, where $Q$ is some cube centered at 0 with edge $C^{\prime}$ depending on $K_{1}, K_{2}, K_{3}, \varepsilon_{1}$, and $\varepsilon_{2}$. Therefore, an argument similar to that of Lemma 1 in [Ca-Gu1] shows that overlapping in each $\mathcal{F}_{k}^{\prime}$ is at most $\alpha$ depending only on $C, C^{\prime}$, and $n$, but not on $k$.

We now prove that $\mathcal{F}_{k}^{\prime}=\left\{\widetilde{Q}\left(z_{i}^{k}, r_{i}^{k}\right)\right\}_{i=1}^{\infty}$ is finite. We set again $z_{i}^{k}=z_{i}$ and $r_{i}^{k}=r_{i}$. Since $O$ is bounded and $M 2^{-(k+1)}<r_{i} \leq M 2^{-k}$, there is a constant $C$ such that $O_{k} \subset \widetilde{Q}\left(z_{1}, C r_{1}\right)$ and $C r_{1} \geq M 2^{-k}$. By (A) we obtain

$$
\begin{gathered}
B\left(x_{i}, K_{2}\left(\frac{r_{i}}{C r_{1}}\right)^{\varepsilon_{2}}\right) \times\left(t_{i}-\frac{r_{i}}{2 C r_{1}}, t_{i}+\frac{r_{i}}{2 C r_{1}}\right) \subset T_{p}\left(\widetilde{Q}\left(z_{i}, r_{i}\right)\right) \\
\subset B\left(x_{i}, K_{1}\left(\frac{r_{i}}{C r_{1}}\right)^{\varepsilon_{1}}\right) \times\left(t_{i}-\frac{r_{i}}{2 C r_{1}}, t_{i}+\frac{r_{i}}{2 C r_{1}}\right),
\end{gathered}
$$

where $T_{p}$ is an affine transformation that normalizes $\widetilde{Q}\left(z_{1}, C r_{1}\right)$, and $\left(x_{i}, t_{i}\right) \in$ $K\left(0, K_{3}\right)$. Therefore

$$
\begin{gathered}
B\left(x_{i}, K_{2}\left(\frac{1}{2 C}\right)^{\varepsilon_{2}}\right) \times\left(t_{i}-\frac{1}{4 C}, t_{i}+\frac{1}{4 C}\right) \subset T_{p}\left(\widetilde{Q}\left(z_{i}, r_{i}\right)\right) \\
\subset B\left(0, K_{1}+K_{3}\right) \times\left(-\left(1+K_{3}^{2}\right), 1+K_{3}^{2}\right) .
\end{gathered}
$$

Since $\mathcal{F}_{k}^{\prime}$ has overlapping bounded by $\alpha$, therefore

$$
\sum_{i} \chi_{T_{p}\left(\widetilde{Q}\left(z_{i}, r_{i}\right)\right)}(z) \leq \alpha
$$

and hence by (2-5)

$$
\sum_{i} \chi_{K\left(z_{i}^{\prime}, d_{1}\right)}(z) \leq \alpha \chi_{K\left(0, d_{2}\right)}
$$

where $d_{1}, d_{2}$ depend only on $K_{1}, K_{2}, K_{3}, \varepsilon_{2}$, and $C$. Integrating (2-6) we get

$$
\sum_{i} \omega_{n} d_{1}^{n+2} \leq \alpha \omega_{n} d_{2}^{n+2}
$$

which implies there are only finite sections in $\mathcal{F}_{k}^{\prime}$. 
Next, we need to estimate the boundedness of overlapping between different generations. To this end, we need to shrink the selected sections. Let $0<\varepsilon<1$ and

$$
z_{0} \in \bigcap_{i} \widetilde{Q}\left(z_{j_{i}}^{e_{i}},(1-\varepsilon) r_{j_{i}}^{e_{i}}\right),
$$

where $e_{1}<e_{2}<\cdots<e_{i}<\cdots, M 2^{-\left(e_{i}+1\right)}<r_{j_{i}}^{e_{i}} \leq M 2^{-e_{i}}$. For simplicity, we set $z_{i}=z_{j_{i}}^{e_{i}}$ and $r_{i}=r_{j_{i}}^{e_{i}}$. Fix $i$ and $l>i$, we need to measure the gap between $e_{i}$ and $e_{l}$. Let $T_{p}$ be an affine transformation that normalizes the section $\widetilde{Q}\left(z_{i}, r_{i}\right)$. Since $r_{i}>r_{l}$, by (A) there exists a point $z^{\prime}=\left(x^{\prime}, t^{\prime}\right) \in K\left(0, K_{3}\right)$ such that

$$
\begin{aligned}
B\left(x^{\prime}, K_{2}\right. & \left.\left(\frac{(1-\varepsilon) r_{l}}{r_{i}}\right)^{\varepsilon_{2}}\right) \times\left(t^{\prime}-\frac{(1-\varepsilon) r_{l}}{2 r_{i}}, t^{\prime}+\frac{(1-\varepsilon) r_{l}}{2 r_{i}}\right) \subset T_{p}\left(\widetilde{Q}\left(z_{l},(1-\varepsilon) r_{l}\right)\right) \\
& \subset B\left(x^{\prime}, K_{1}\left(\frac{(1-\varepsilon) r_{l}}{r_{i}}\right)^{\varepsilon_{1}}\right) \times\left(t^{\prime}-\frac{(1-\varepsilon) r_{l}}{2 r_{i}}, t^{\prime}+\frac{(1-\varepsilon) r_{l}}{2 r_{i}}\right) .
\end{aligned}
$$

Since by construction $z_{l} \notin \widetilde{Q}\left(z_{i}, r_{i}\right)$, by (B) we have that

$$
K\left(T_{p}\left(z_{l}\right), \varepsilon^{\delta}\right) \cap T_{p}\left(\widetilde{Q}\left(z_{i},(1-\varepsilon) r_{i}\right)\right)=\emptyset .
$$

It follows that

$$
\begin{aligned}
\frac{1}{2} \varepsilon^{2 \delta} & <\left|T_{p}\left(z_{l}\right)-T_{p}\left(z_{0}\right)\right| \\
& \leq\left|T_{p}\left(z_{l}\right)-z^{\prime}\right|+\left|z^{\prime}-T_{p}\left(z_{0}\right)\right| \\
& \leq 2\left[K_{1}\left(\frac{(1-\varepsilon) r_{l}}{r_{i}}\right)^{\varepsilon_{1}}+\frac{(1-\varepsilon) r_{l}}{2 r_{i}}\right] \\
& \leq 2\left[K_{1} 2^{\left(e_{i}+1-e_{l}\right) \varepsilon_{1}}+2^{e_{i}-e_{l}}\right],
\end{aligned}
$$

which implies that $e_{l}-e_{i} \leq \bar{C} \log (1 / \varepsilon)$, where $\bar{C}$ depends on $\delta, \varepsilon_{1}$ and $K_{1}$. Therefore, the number of sections in (2-7) is at most $\bar{C} \log (1 / \varepsilon)$.

We are now in a position to show that the collection $\mathcal{F}^{\prime}$ of all $\mathcal{F}_{k}^{\prime}$ satisfies properties (i)-(iii) in Lemma 2.1. Since each $\mathcal{F}_{k}^{\prime}$ has only finite elements, by our construction, $\mathcal{F}_{k}^{\prime}$ covers $O_{k}$ and thus $\mathcal{F}^{\prime}$ covers $O$. By our construction and the fact that $\mathcal{F}_{k}^{\prime}$ is a finite set, relabelling the sections in $\mathcal{F}^{\prime}$, (ii) is automatically satisfied. By the argument above on boundedness of overlapping between sections, it is easy to check that (iii) is valid.

In the following, we will develop a variant form of Calderón-Zygmund decomposition in terms of parabolic sections. Let us first mention the following lemma due to [Ca-Gu2].

Lemma 2.2. Let $\mu$ be the Monge-Ampère measure generated by $\phi(x)$. Suppose that $\mu$ satisfies (1-2) and (1-3). Then there exist constants $C_{1}>0$ and $0<\theta_{0}<1$ such that for any section $S$ and any measurable subset $E \subset S$

$$
\frac{\mu(E)}{\mu(S)} \leq C_{1}\left(\frac{|E|}{|S|}\right)^{\theta_{0}}
$$

where $|E|$ denotes Lebesgue measure of $E$.

The following Calderón-Zygmund decomposition is a generalization of Theorem 3 in [Ca-Gu2]. 
Theorem 2.1. Let $\mu$ be the Monge-Ampère measure generated by $\phi(x)$, and $d \mathcal{M}=$ $d \mu d t$ be the parabolic Monge-Ampère measure generated by $\phi(x)-t$. Suppose that $\mu$ satisfies (1-2) and (1-3). Given a bounded open set $O$ and $0<\lambda<1$, there exists a family of parabolic sections $\mathcal{F}=\left\{\widetilde{Q}\left(z_{k}, r_{k}\right)\right\}_{k=1}^{\infty}$ with the following properties:

(i) $z_{k} \in O, \forall k$.

(ii) $O \subset \bigcup_{k=1}^{\infty} \widetilde{Q}\left(z_{k}, r_{k}\right)$.

(iii) $\frac{\mathcal{M}\left(O \cap \widetilde{Q}\left(z_{k}, r_{k}\right)\right)}{\mathcal{M}\left(\widetilde{Q}\left(z_{k}, r_{k}\right)\right)}=\lambda$.

(iv) $\mathcal{M}(O)<c(\lambda) \mathcal{M}\left(\bigcup_{k=1}^{\infty} \widetilde{Q}\left(z_{k}, r_{k}\right)\right)$, where $0<c(\lambda)<1$ is a constant depending only on $\lambda$ but not on $O$ nor $\mathcal{F}$.

Proof. The proof is similar to that of Theorem 3 in [Ca-Gu2]. First observe (see [Ca-Gu2])

$$
|S(x, r)|-|S(x,(1-\varepsilon) r)| \leq n \varepsilon|S(x, r)| .
$$

By Lemma 2.2

$$
\mu(S(x, r))-\mu(S(x,(1-\varepsilon) r)) \leq C \varepsilon^{\theta_{0}} \mu(S(x, r)) .
$$

Therefore

$$
\begin{aligned}
& \mathcal{M}(\widetilde{Q}(z, r))-\mathcal{M}(\widetilde{Q}(z,(1-\varepsilon) r)) \\
= & r \mu(S(x, r))-(1-\varepsilon) r \mu(S(x,(1-\varepsilon) r)) \\
= & \varepsilon r \mu(S(x, r))+(1-\varepsilon) r(\mu(S(x, r))-\mu(S(x,(1-\varepsilon) r))) \\
\leq & \varepsilon r \mu(S(x, r))+C \varepsilon^{\theta_{0}} \mu(S(x, r))(1-\varepsilon) r \\
\leq & 2 C \varepsilon^{\theta_{0}} \mathcal{M}(\widetilde{Q}(z, r)) .
\end{aligned}
$$

For $z \in O$, consider the average

$$
a(r)=\frac{\mathcal{M}(O \cap \widetilde{Q}(z, r))}{\mathcal{M}(\widetilde{Q}(z, r))}
$$

Obviously, $a(r)$ is continuous, $\lim _{r \rightarrow \infty} a(r)=0$, and $a(r)=1$ if $r$ is sufficiently small. For $0<\lambda<1$, let

$$
r_{z}=\sup \{r: a(r) \geq \lambda\} .
$$

Then we have

$$
\frac{\mathcal{M}\left(O \cap \widetilde{Q}\left(z, r_{z}\right)\right)}{\mathcal{M}\left(\widetilde{Q}\left(z, r_{z}\right)\right)}=\lambda,
$$

and for $r^{\prime}>r_{z}$

$$
\frac{\mathcal{M}\left(O \cap \widetilde{Q}\left(z, r^{\prime}\right)\right)}{\mathcal{M}\left(\widetilde{Q}\left(z, r^{\prime}\right)\right)}<\lambda .
$$

Note that $\mu(S(x, 2 r)) \geq C \mu(S(x, r))$ with $C>1$ and therefore

$$
\mathcal{M}(\widetilde{Q}(z, 2 r)) \geq C^{\prime} \mathcal{M}(\widetilde{Q}(z, r)), \quad C^{\prime}>1 .
$$

From (2-9) and the boundedness of $O, \mathcal{M}\left(\widetilde{Q}\left(z, r_{z}\right)\right)$ is bounded. Take $z_{0} \in O$ and $r_{0}$ sufficiently large such that $O \subset \widetilde{Q}\left(z_{0}, r_{0}\right)$. By the engulfing property, $\widetilde{Q}\left(z_{0}, r_{0}\right) \subset$ $\widetilde{Q}\left(z, \theta r_{0}\right)$ for $z \in O$. Therefore, by (2-11), it is easy to show that $r_{z}$ is bounded. 
Now consider $\mathcal{F}=\left\{\widetilde{Q}\left(z, r_{z}\right)\right\}_{z \in O}$. Apply Lemma 2.1 to $\mathcal{F}$ and obtain a countable subfamily of $\mathcal{F}$ denoted by $\left\{\widetilde{Q}\left(z_{k}, r_{k}\right)\right\}_{k=1}^{\infty}$. Clearly, $\left\{\widetilde{Q}\left(z_{k}, r_{k}\right)\right\}_{k=1}^{\infty}$ satisfies properties (i)-(iii) in Theorem 2.1 by Lemma 2.1 and (2-9). It remains to show (iv) in Theorem 2.1. We adopt some notations. Let $\widetilde{Q}_{k}^{\varepsilon}=\widetilde{Q}\left(z_{k},(1-\varepsilon) r_{k}\right)$ and $\widetilde{Q}_{k}=\widetilde{Q}\left(z_{k}, r_{k}\right)$ for $k \geq 1$. Let $f(z)$ be the overlapping function defined by

$$
f(z)= \begin{cases}\#\left\{k: z \in \widetilde{Q}_{k}\right\}, & \text { if } z \in \bigcup \widetilde{Q}_{k}, \\ 1, & \text { if } z \notin \bigcup \widetilde{Q}_{k} .\end{cases}
$$

Another overlapping function $f_{\varepsilon}(z)$ can be defined similarly. By Lemma $2.1,1 \leq$ $f_{\varepsilon}(z) \leq C_{0} \log (1 / \varepsilon)$. Since $f(z)$ may be infinite, in the following argument, we work with any finite family $\left\{\widetilde{Q}_{k}\right\}_{k=1}^{N}$ and then let $N \rightarrow \infty$. Obviously

$$
\begin{aligned}
\mathcal{M}(O) & =\mathcal{M}\left(\left(\bigcup \widetilde{Q}_{k}\right) \cap O\right) \\
& =\int \frac{1}{f(z)} \sum_{k} \chi_{\widetilde{Q}_{k} \cap O}(z) d \mathcal{M} \\
& \leq \sum_{k} \int \frac{1}{f_{\varepsilon}(z)} \chi_{\widetilde{Q}_{k} \cap O}(z) d \mathcal{M} .
\end{aligned}
$$

By (2-9), we then have

$$
\begin{aligned}
\int \frac{1}{f_{\varepsilon}(z)} \chi_{\widetilde{Q}_{k} \backslash O}(z) d \mathcal{M} & \geq \frac{1}{C_{0} \log (1 / \varepsilon)} \mathcal{M}\left(\widetilde{Q}_{k} \backslash O\right) \\
& =\frac{1-\lambda}{C_{0} \log (1 / \varepsilon)} \mathcal{M}\left(\widetilde{Q}_{k}\right) \\
& \geq \frac{1-\lambda}{C_{0} \log (1 / \varepsilon)} \int \frac{1}{f_{\varepsilon}(z)} \chi_{\widetilde{Q}_{k}}(z) d \mathcal{M}
\end{aligned}
$$

Therefore

$$
\begin{aligned}
& \int \frac{1}{f_{\varepsilon}(z)} \chi_{\widetilde{Q}_{k} \cap O}(z) d \mathcal{M} \\
= & \int \frac{1}{f_{\varepsilon}(z)}\left(\chi_{\widetilde{Q}_{k}(z)}-\chi_{\widetilde{Q}_{k} \backslash O}(z)\right) d \mathcal{M} \\
\leq & \left(1-\frac{1-\lambda}{C_{0} \log (1 / \varepsilon)}\right) \int \frac{1}{f_{\varepsilon}(z)} \chi_{\widetilde{Q}_{k}}(z) d \mathcal{M} .
\end{aligned}
$$

It follows from (2-12) that

$$
\mathcal{M}(O) \leq\left(1-\frac{1-\lambda}{C_{0} \log (1 / \varepsilon)}\right) \sum_{k} \int \frac{1}{f_{\varepsilon}(z)} \chi_{\widetilde{Q}_{k}}(z) d \mathcal{M}
$$

By $(2-8)$, we have

$$
\mathcal{M}(\widetilde{Q}(z, r))-\mathcal{M}(\widetilde{Q}(z,(1-\varepsilon) r)) \leq \frac{2 C \varepsilon^{\theta_{0}}}{1-2 C \varepsilon^{\theta_{0}}} \mathcal{M}(\widetilde{Q}(z,(1-\varepsilon) r)) .
$$


Noting $1 \leq f_{\varepsilon} \leq C_{0} \log (1 / \varepsilon)$, we then have

$$
\begin{aligned}
& \int \frac{1}{f_{\varepsilon}(z)} \chi_{\widetilde{Q}_{k}}(z) d \mathcal{M} \\
\leq & \mathcal{M}\left(\widetilde{Q}_{k}\right)-\mathcal{M}\left(\widetilde{Q}_{k}^{\varepsilon}\right)+\int \frac{1}{f_{\varepsilon}(z)} \chi_{\widetilde{Q}_{k}^{\varepsilon}}(z) d \mathcal{M} \\
\leq & \frac{2 C \varepsilon^{\theta_{0}}}{1-2 C \varepsilon^{\theta_{0}}} \mathcal{M}\left(\widetilde{Q}_{k}^{\varepsilon}\right)+\int \frac{1}{f_{\varepsilon}(z)} \chi_{\widetilde{Q}_{k}^{\varepsilon}}(z) d \mathcal{M} \\
\leq & \left(\frac{2 C \varepsilon^{\theta_{0}}}{1-2 C \varepsilon^{\theta_{0}}} C_{0} \log (1 / \varepsilon)+1\right) \int \frac{1}{f_{\varepsilon}(z)} \chi_{\widetilde{Q}_{k}^{\varepsilon}}(z) d \mathcal{M} .
\end{aligned}
$$

By adding over $k$ in the above inequality, we get

$$
\begin{aligned}
\mathcal{M}(O) \leq & \left(1-\frac{1-\lambda}{C_{0} \log (1 / \varepsilon)}\right)\left(\frac{2 C \varepsilon^{\theta_{0}}}{1-2 C \varepsilon^{\theta_{0}}} C_{0} \log (1 / \varepsilon)+1\right) \\
& \times \sum_{k} \int \frac{1}{f_{\varepsilon}(z)} \chi_{\widetilde{Q}_{k}^{\varepsilon}}(z) d \mathcal{M} \\
= & \left(1-\frac{1-\lambda}{C_{0} \log (1 / \varepsilon)}\right)\left(\frac{2 C \varepsilon^{\theta_{0}}}{1-2 C \varepsilon^{\theta_{0}}} C_{0} \log (1 / \varepsilon)+1\right) \mathcal{M}\left(\bigcup \widetilde{Q}_{k}^{\varepsilon}\right) \\
= & g(\varepsilon) \mathcal{M}\left(\bigcup \widetilde{Q}_{k}^{\varepsilon}\right) .
\end{aligned}
$$

Obviously

$$
\begin{aligned}
g(\varepsilon) & <1+\frac{2 C \varepsilon^{\theta_{0}}}{1-2 C \varepsilon^{\theta_{0}}} C_{0} \log (1 / \varepsilon)-\frac{1-\lambda}{C_{0} \log (1 / \varepsilon)} \\
& =1-\frac{1-\lambda}{C_{0} \log (1 / \varepsilon)}\left(1-\frac{2 C \varepsilon^{\theta_{0}}}{1-2 C \varepsilon_{\theta_{0}}} \cdot \frac{\left(C_{0} \log (1 / \varepsilon)\right)^{2}}{1-\lambda}\right) .
\end{aligned}
$$

Therefore, for $0<\lambda<1$, there exists $\varepsilon_{0}$ such that $g(\varepsilon)<1$ if $\varepsilon<\varepsilon_{0}$. So, for $0<\lambda<1$, we can have $0<c(\lambda)<1$ such that $\mathcal{M}(O)<c(\lambda) \mathcal{M}\left(\bigcup \widetilde{Q}_{k}\right)$.

Theorem 2.1 cannot be directly used in discussing the power decay of the distribution function, since there is a shift in time for estimates for parabolic equations. In the following, we will give another form of the Calderón-Zygmund decomposition. Let us first show an elementary lemma.

For $k=1, \cdots, N$, let $\Omega_{k}$ be a convex set in $\mathbf{R}^{n}$ and $A_{k}=\Omega_{k} \times\left[a_{k}, b_{k}\right]$ be a cylinder in $\mathbf{R}^{n+1}$. Let $m \geq 1$ be an integer. Set

$$
\begin{aligned}
& A_{k}^{(i)}=\Omega_{k} \times\left[a_{k}+(i-1)\left(b_{k}-a_{k}\right), a_{k}+i\left(b_{k}-a_{k}\right)\right], \\
& \bar{A}_{k}^{m}=\bigcup_{i=1}^{m+1} A_{k}^{(i)}=\Omega_{k} \times\left[a_{k}, a_{k}+(m+1)\left(b_{k}-a_{k}\right)\right], \\
& A_{k}^{m}=\bigcup_{i=2}^{m+1} A_{k}^{(i)}=\Omega_{k} \times\left[b_{k}, a_{k}+(m+1)\left(b_{k}-a_{k}\right)\right] .
\end{aligned}
$$

Lemma 2.3. Let $\mu$ be a $\sigma$-finite Borel measure in $\mathbf{R}^{n}$ and $\mathcal{M}=\mu \times d t$. Here $d t$ is the Lebesgue measure in $\mathbf{R}^{1}$. Then

$$
\mathcal{M}\left(\bigcup_{k=1}^{N} \bar{A}_{k}^{m}\right) \leq \frac{m+1}{m} \mathcal{M}\left(\bigcup_{k=1}^{N} A_{k}^{m}\right)
$$


Proof. We show this lemma by inductive argument. In case $N=1$, obviously

$$
\mathcal{M}\left(\bar{A}_{1}^{m}\right)=\frac{m+1}{m} \mathcal{M}\left(A_{1}^{m}\right) .
$$

In case $N=2$, clearly

$$
\mathcal{M}\left(\bar{A}_{1}^{m} \cup \bar{A}_{2}^{m}\right)=\mathcal{M}\left(\bar{A}_{1}^{m}-\bar{A}_{2}^{m}\right)+\mathcal{M}\left(\bar{A}_{2}^{m}\right) .
$$

Therefore

$$
\begin{aligned}
\mathcal{M}\left(\bar{A}_{1}^{m} \cup \bar{A}_{2}^{m}\right) & \leq \mathcal{M}\left(\bar{A}_{1}^{m}-\bar{A}_{2}^{m}\right)+\frac{m+1}{m} \mathcal{M}\left(A_{2}^{m}\right) \\
& =\mathcal{M}\left(A_{1}-\bar{A}_{2}^{m}\right)+\mathcal{M}\left(A_{1}^{m}-\bar{A}_{2}^{m}\right)+\frac{m+1}{m} \mathcal{M}\left(A_{2}^{m}\right) .
\end{aligned}
$$

Without loss of generality, we may assume that $a_{2} \leq a_{1}$. Since Lebesgue measure $d t$ is invariant under translation, $a_{2} \leq a_{1}$, and by the structure of $A_{1}^{(i)}$ and $\bar{A}_{2}^{m}$, it is easy to check

$$
\mathcal{M}\left(A_{1}-\bar{A}_{2}^{m}\right) \leq \mathcal{M}\left(A_{1}^{(i)}-\bar{A}_{2}^{m}\right), \quad \text { for } 2 \leq i \leq m+1 .
$$

Summing (2-15) over $i$ yields

$$
m \mathcal{M}\left(A_{1}-\bar{A}_{2}^{m}\right) \leq \mathcal{M}\left(A_{1}^{m}-\bar{A}_{2}^{m}\right) .
$$

It follows that

$$
\begin{aligned}
\mathcal{M}\left(\bar{A}_{1}^{m} \cup \bar{A}_{2}^{m}\right) & \leq\left(\frac{1}{m}+1\right) \mathcal{M}\left(A_{1}^{m}-\bar{A}_{2}^{m}\right)+\frac{m+1}{m} \mathcal{M}\left(A_{2}^{m}\right) \\
& \leq \frac{m+1}{m}\left(\mathcal{M}\left(A_{1}^{m}-A_{2}^{m}\right)+\mathcal{M}\left(A_{2}^{m}\right)\right) \\
& =\frac{m+1}{m} \mathcal{M}\left(A_{1}^{m} \cup A_{2}^{m}\right) .
\end{aligned}
$$

Suppose that (2-14) is true for case $N$.

In case $N+1$, by relabelling the indices, we may assume that $a_{k} \leq a_{1}$ for $k \geq 2$. Clearly

$$
\begin{aligned}
\mathcal{M}\left(\bar{A}_{1}^{m} \cup\right. & \left.\cdots \cup \bar{A}_{N+1}^{m}\right) \leq \mathcal{M}\left(A_{1}-\left(\bar{A}_{2}^{m} \cup \cdots \cup \bar{A}_{N+1}^{m}\right)\right) \\
& +\mathcal{M}\left(A_{1}^{m}-\left(\bar{A}_{2}^{m} \cup \cdots \cup \bar{A}_{N+1}^{m}\right)\right)+\mathcal{M}\left(\bar{A}_{2}^{m} \cup \cdots \cup \bar{A}_{N+1}^{m}\right) .
\end{aligned}
$$

Analogous to (2-15), we have for $2 \leq i \leq m+1$

$$
\mathcal{M}\left(A_{1}-\left(\bar{A}_{2}^{m} \cup \cdots \cup \bar{A}_{N+1}^{m}\right)\right) \leq \mathcal{M}\left(A_{1}^{(i)}-\left(\bar{A}_{2}^{m} \cup \cdots \cup \bar{A}_{N+1}^{m}\right)\right) .
$$

Therefore, it is easy to see by inductive hypothesis that

$$
\begin{aligned}
& \mathcal{M}\left(\bar{A}_{1}^{m} \cup \cdots \cup \bar{A}_{N+1}^{m}\right) \\
\leq & \left(1+\frac{1}{m}\right) \mathcal{M}\left(A_{1}^{m}-\left(\bar{A}_{2}^{m} \cup \cdots \cup \bar{A}_{N+1}^{m}\right)\right)+\frac{m+1}{m} \mathcal{M}\left(A_{2}^{m} \cup \cdots \cup A_{N+1}^{m}\right) \\
\leq & \frac{m+1}{m} \mathcal{M}\left(A_{1}^{m} \cup \cdots \cup A_{N+1}^{m}\right) .
\end{aligned}
$$

The proof is completed. 
By Theorem 2.1 and Lemma 2.3, it follows that

$$
\begin{aligned}
\mathcal{M}(O) & <c(\lambda) \mathcal{M}\left(\bigcup_{k=1}^{\infty} \widetilde{Q}\left(z_{k}, r_{k}\right)\right) \\
& \leq c(\lambda) \mathcal{M}\left(\bigcup_{k=1}^{\infty} \widetilde{\widetilde{Q}}^{m}\left(z_{k}, r_{k}\right)\right) \\
& \leq c(\lambda) \frac{m+1}{m} \mathcal{M}\left(\bigcup_{k=1}^{\infty} \widetilde{Q}^{m}\left(z_{k}, r_{k}\right)\right),
\end{aligned}
$$

where

$$
\begin{aligned}
& \widetilde{\widetilde{Q}}^{m}\left(z_{k}, r_{k}\right)=S\left(x_{k}, r_{k}\right) \times\left[t_{k}-\frac{r_{k}}{2}, t_{k}+\left(m+\frac{1}{2}\right) r_{k}\right], \\
& \widetilde{Q}^{m}\left(z_{k}, r_{k}\right)=S\left(x_{k}, r_{k}\right) \times\left[t_{k}+\frac{r_{k}}{2}, t_{k}+\left(m+\frac{1}{2}\right) r_{k}\right] .
\end{aligned}
$$

Therefore we conclude the following variant of Calderón-Zygmund decomposition which generalizes a result in $[\mathrm{W}]$.

Theorem 2.2. Suppose that all assumptions of Theorem 2.1 hold. Given a bounded open set $O$ and $0<\lambda<1$, there exists a family of parabolic sections $\mathcal{F}=$ $\left\{\widetilde{Q}\left(z_{k}, r_{k}\right)\right\}_{k=1}^{\infty}$ with the following properties:

(i) $z_{k} \in O, \quad \forall k$.

(ii) $O \subset \bigcup_{k=1}^{\infty} \widetilde{Q}\left(z_{k}, r_{k}\right)$.

(iii) $\frac{\mathcal{M}\left(O \cap \widetilde{Q}\left(z_{k}, r_{k}\right)\right)}{\mathcal{M}\left(\widetilde{Q}\left(z_{k}, r_{k}\right)\right)}=\lambda$.

(iv) $\mathcal{M}(O)<c(\lambda) \frac{m+1}{m} \mathcal{M}\left(\bigcup_{k=1}^{\infty} \widetilde{Q}^{m}\left(z_{k}, r_{k}\right)\right)$, for any $m \geq 1$, where $0<c(\lambda)<1$ depends on $\lambda$ but not on $O$ or $\mathcal{F}$.

\section{Density of the Distribution Function}

In this section, we will give estimates about the density of the distribution function of solutions of (1-1).

For $z_{0}=\left(x_{0}, t_{0}\right)$, let $Q\left(z_{0}, r\right)=S\left(x_{0}, r\right) \times\left(t_{0}-r, t_{0}\right]$ be a section. Let $T$ be an affine transformation that normalizes $S\left(x_{0}, r\right)$ and $T_{p}$ an affine transformation normalizing $Q\left(z_{0}, r\right)$ defined by

$$
T_{p}:(x, t) \rightarrow\left(T x, \frac{t-\left(t_{0}-r\right)}{r}\right) .
$$

Therefore

$$
B\left(0, \alpha_{n}\right) \times(0,1] \subset T_{p}\left(Q\left(z_{0}, r\right)\right) \subset B(0,1) \times(0,1]
$$

In the following, we introduce the notions of normalizations of sections and of the functions. Set

$$
\psi_{r}(x)=\frac{1}{r} \phi\left(T^{-1} x\right)
$$

and

$$
u^{*}(z)=u\left(T_{p}^{-1} z\right)=u\left(T^{-1} x, t_{0}-r+r t\right)
$$


It is easy to check that

$$
\begin{aligned}
& S^{*}=T\left(S_{\phi}\left(x_{0}, r\right)\right)=S_{\psi_{r}}\left(T x_{0}, 1\right), \\
& Q^{*}=T_{p}\left(Q_{\phi}\left(z_{0}, r\right)\right)=Q_{\psi_{r}}\left(T_{p}\left(z_{0}\right), 1\right)=S^{*} \times(0,1] .
\end{aligned}
$$

Let $u$ be a solution of (1-1). Note that

$$
\begin{aligned}
& D^{2} \psi_{r}(x)=\frac{1}{r}\left(J^{-1}\right)^{t} D^{2} \phi\left(T^{-1} x\right) J^{-1}, \\
& D^{2} u^{*}(z)=\left(J^{-1}\right)^{t} D^{2} u\left(T_{p}^{-1} z\right) J^{-1}, \\
& u_{t}^{*}(z)=r u_{s}\left(T_{p}^{-1} z\right),
\end{aligned}
$$

where $J$ denotes the Jacobian of $T$. Hence (1-1) becomes under $T_{p}$

$$
\frac{1}{r} u_{t}^{*}-\operatorname{tr}\left(\frac{1}{r} J^{-1}\left(D^{2} \psi_{r}\right)^{-1}\left(J^{-1}\right)^{t} J^{t} D^{2} u^{*} J\right)=0,
$$

and after simplifying $u^{*}$ satisfies the following equation:

$$
u_{t}^{*}-\operatorname{tr}\left(\left(D^{2} \psi_{r}\right)^{-1} D^{2} u^{*}\right)=0 .
$$

Since $T$ normalizes $S_{\phi}\left(x_{0}, r\right)$ and hence $\left|T S_{\phi}\left(x_{0}, r\right)\right| \approx C_{n}$, we have

$$
|\operatorname{det} J| \cdot\left|S_{\phi}\left(x_{0}, r\right)\right| \approx C_{n} .
$$

Let $\mu^{*}$ denote the Monge-Ampère measure generated by $\psi_{r}$. By (3-4)

$$
d \mu^{*}=r^{-n}(\operatorname{det} J)^{-2} \operatorname{det} D^{2} \phi\left(T^{-1} x\right) d x .
$$

It easily follows that

$$
\begin{aligned}
\mu^{*}\left(S^{*}\right) & =\int_{T\left(S_{\phi}\left(x_{0}, r\right)\right)} r^{-n}(\operatorname{det} J)^{-2} \operatorname{det} D^{2} \phi\left(T^{-1} x\right) d x \\
& =\int_{S_{\phi}\left(x_{0}, r\right)} r^{-n}|\operatorname{det} J|^{-1} \operatorname{det} D^{2} \phi(x) d x \\
& =r^{-n}|\operatorname{det} J|^{-1} \mu\left(S_{\phi}\left(x_{0}, r\right)\right) .
\end{aligned}
$$

On the other hand, since $\mu$ satisfies doubling condition (1-2), $\mu^{*}$ also satisfies (1-2). We define the normalization $\phi^{*}$ of $\phi$ by

$$
\phi^{*}(x)=\psi_{r}(x)-\bar{\ell}_{T x_{0}}(x)-1,
$$

where $\bar{\ell}_{T x_{0}}(x)$ is the supporting hyperplane of $\psi_{r}$ at $T x_{0}$. Obviously, the MongeAmpère measure generated by $\phi^{*}$ is exactly $\mu^{*}, \phi^{*}=0$ on $\partial S^{*}$, and $\max _{S^{*}}\left|\phi^{*}\right|=1$. By Lemma 1.1 in [Ca-Gu2] or [Ca1], we have that $\mu^{*}\left(S^{*}\right) \approx C_{n} \max _{S^{*}}\left|\phi^{*}\right|^{n} \approx C_{n}$, i.e.,

$$
r^{n}|\operatorname{det} J| \approx C_{n} \mu\left(S_{\phi}\left(x_{0}, r\right)\right) .
$$

We are ready to give estimates about the distribution function of the solution $u$ of (1-1).

Lemma 3.1. Let $u \geq 0$ be a classical solution of (1-1) in $Q\left(z_{0}, r\right)$. Suppose that

$$
\inf \left\{u(z): z \in Q\left(z_{0}, r / 2\right)\right\} \leq 1 .
$$

Then there exist $\varepsilon_{0} \in(0,1), M_{0}>1$ such that

$$
\mathcal{M}\left(\left\{z \in Q\left(z_{0}, r\right): u(z)<M_{0}\right\}\right)>\varepsilon_{0} \mathcal{M}\left(Q\left(z_{0}, r\right)\right),
$$

where $\varepsilon_{0}$ and $M_{0}$ depend only on $n$ and the structure conditions of $\phi$, and are independent of $u$. 
Proof. By the previous argument, $u^{*}(z)$ satisfies $(3-5)$ in $Q^{*} ; \phi^{*}(x)=0$ on $\partial S^{*}$; $-1 \leq \phi^{*}(x) \leq 0$ in $S^{*} ;-1 \leq \phi^{*}(x) \leq-\frac{1}{2}$ in $S_{1 / 2}^{*}=T\left(S_{\phi}\left(x_{0}, r / 2\right)\right)=S_{\psi_{r}}\left(T x_{0}, \frac{1}{2}\right)$. Consider the auxiliary function

$$
w(z)=u^{*}(z)+16 t \phi^{*}(x) .
$$

We may assume that

$$
u^{*}\left(z^{\prime}\right)=\inf \left\{u^{*}(z): z \in S_{1 / 2}^{*} \times\left(\frac{1}{2}, 1\right)=Q_{1 / 2}^{*}\right\} \leq 1,
$$

where $z^{\prime} \in \overline{Q_{1 / 2}^{*}}$.

Let $\Gamma\left(w^{-}\right)$denote the parabolic concave envelope in $Q^{*}$ of the negative part $w^{-}$ of $w$ and $A_{w}$ be the contact set, i.e.,

$$
A_{w}=\left\{z \in Q^{*}: w<0, w=-\Gamma\left(w^{-}\right)\right\} .
$$

It was proved in $[\mathrm{W}]$ that $\Gamma\left(w^{-}\right)$is $C^{1,1}$ and $\left(\sup _{Q^{*}} w^{-}\right)^{n+1}$ is controlled by the volume of the image of $A_{w}$ under the transformation

$$
z \rightarrow\left(D \Gamma\left(w^{-}\right), \Gamma\left(w^{-}\right)-x D \Gamma\left(w^{-}\right)\right) .
$$

By $[T]$, we have

$$
w^{-}\left(z^{\prime}\right)^{n+1} \leq C\left(\operatorname{diam}\left(S^{*}\right)\right)^{n} \iint_{A_{w}}\left|\Gamma\left(w^{-}\right)_{t} \operatorname{det} D^{2}\left(\Gamma\left(w^{-}\right)\right)\right| d x d t .
$$

Obviously, $w \geq-\Gamma\left(w^{-}\right)$on $Q^{*}$. It is easy to check that on $A_{w}$

$$
\left\{\begin{array}{l}
D^{2} w \geq D^{2}\left(-\Gamma\left(w^{-}\right)\right) \geq 0, \\
w_{t} \leq\left(-\Gamma\left(w^{-}\right)\right)_{t} \leq 0
\end{array}\right.
$$

It follows from (3-10) that

$$
1 \leq C \iint_{A_{w}}\left(-w_{t}\right) \operatorname{det} D^{2} w d x d t
$$

By the geometric-arithmetic mean inequality, we have the following estimate on $A_{w}$ :

$$
\begin{aligned}
-w_{t} \operatorname{det} D^{2} w & \leq\left[-w_{t}+\operatorname{tr}\left(\left(D^{2} \phi^{*}\right)^{-1} D^{2} w\right)\right]^{n+1}(n+1)^{-(n+1)} \operatorname{det} D^{2} \phi^{*} \\
& =\left(-L_{\psi_{r}} w\right)^{n+1}(n+1)^{-(n+1)} \operatorname{det} D^{2} \psi_{r} \\
& =\left(-16 \phi^{*}(x)+16 t n\right)^{n+1}(n+1)^{-(n+1)} \operatorname{det} D^{2} \psi_{r} \\
& \leq C_{n} \operatorname{det} D^{2} \psi_{r} .
\end{aligned}
$$

Noting that $A_{w} \subset\left\{z \in Q^{*}: u^{*}(z)<17\right\}$, from (3-11), it follows that

$$
\begin{aligned}
1 & \leq C \iint_{\left\{z \in Q^{*}: u^{*}<17\right\}} \operatorname{det} D^{2} \psi_{r} d x d t \\
& =C \iiint_{\left\{z \in Q\left(z_{0}, r\right): u<17\right\}} r^{-n-1}|\operatorname{det} J|^{-1} \operatorname{det} D^{2} \phi d x d t,
\end{aligned}
$$


where we have used a change of variables and the definition of $T_{p}$. By (3-9) we proceed to the following:

$$
\begin{aligned}
1 & \leq C\left(r^{n}|\operatorname{det} J| \cdot r\right)^{-1} \iint_{\left\{z \in Q\left(z_{0}, r\right): u<17\right\}} \operatorname{det} D^{2} \phi d x d t \\
& =C \frac{\mathcal{M}\left(\left\{z \in Q\left(z_{0}, r\right): u(z)<17\right\}\right)}{\mu\left(S_{\phi}\left(x_{0}, r\right)\right) r} .
\end{aligned}
$$

Since $\mu\left(S_{\phi}\left(x_{0}, r\right)\right) r=\mathcal{M}\left(Q\left(z_{0}, r\right)\right)$, the proof of Lemma 3.1 is complete.

We have the following useful corollary of Lemma 3.1.

Lemma 3.2. Let $\varepsilon_{0}$ and $M_{0}$ be the constants in Lemma 3.1. Let $u \geq 0$ be a solution of (1-1) in $S\left(x_{0}, r\right) \times\left(t_{0}-r, t_{0}+\tau r\right]$ with $\tau=\varepsilon_{0} / 2$. Suppose that

$$
\inf \left\{u(z): z \in S\left(x_{0}, r / 2\right) \times\left(t_{0}, t_{0}+\tau r\right]\right\} \leq 1 .
$$

Then

$$
\mathcal{M}\left(\left\{z \in Q\left(z_{0}, r\right): u(z)<M_{0}\right\}\right)>\frac{\varepsilon_{0}}{2} \mathcal{M}\left(Q\left(z_{0}, r\right)\right) .
$$

Proof. Obviously, (3-12) implies that $\inf \left\{u(z): z \in Q\left(\left(x_{0}, t_{0}+\tau r\right), r / 2\right)\right\} \leq 1$. Therefore, by Lemma 3.1, we have

$$
\mathcal{M}\left(\left\{z \in Q\left(\left(x_{0}, t_{0}+\tau r\right), r\right): u(z)<M_{0}\right\}\right)>\varepsilon_{0} \mathcal{M}\left(Q\left(\left(x_{0}, t_{0}+\tau r\right), r\right)\right) .
$$

It immediately follows that

$$
\begin{aligned}
\mathcal{M}\left(\left\{z \in S\left(x_{0}, r\right) \times\left(t_{0}-(1-\tau) r,\right.\right.\right. & \left.\left.\left.t_{0}\right): u(z)<M_{0}\right\}\right) \\
& +\tau r \mu\left(S\left(x_{0}, r\right)\right)>\varepsilon_{0} r \mu\left(S\left(x_{0}, r\right)\right) .
\end{aligned}
$$

Thus

$$
\begin{aligned}
& \mathcal{M}\left(\left\{z \in Q\left(z_{0}, r\right): u(z)<M_{0}\right\}\right) \\
\geq & \mathcal{M}\left(\left\{z \in S\left(x_{0}, r\right) \times\left(t_{0}-(1-\tau) r, t_{0}\right): u(z)<M_{0}\right\}\right) \\
> & \left(\varepsilon_{0} r-\tau r\right) \mu\left(S\left(x_{0}, r\right)\right)=\frac{\varepsilon_{0}}{2} \mathcal{M}\left(S\left(x_{0}, r\right)\right) .
\end{aligned}
$$

By Lemma 3.2, if $u(z) \geq M_{0}$ on $Q\left(z_{0}, r\right)$, then $u(z) \geq 1$ on $S\left(x_{0}, \frac{r}{2}\right) \times\left(t_{0}, t_{0}+\tau r\right]$. This gives us the estimate in the $t$-direction for solutions of (1-1). In the following, we need to consider the estimate in the $x$-direction.

Let $r_{0}=4 r$ and $K>\sigma>\beta>0, \beta<\frac{1}{2}$. We introduce the following notations:

$$
\begin{aligned}
& Q_{4}=S_{\phi}\left(x_{0}, 4 r\right) \times\left(t_{0}-4 K r, t_{0}\right], \\
& Q_{3}=S_{\phi}\left(x_{0}, 4(1-\beta) r\right) \times\left(t_{0}-4(K-\beta) r, t_{0}\right], \\
& Q_{2}=S_{\phi}\left(x_{0}, 2 r\right) \times\left(t_{0}-4(K-\sigma) r, t_{0}\right], \\
& Q_{1}=S_{\phi}\left(x_{0}, r\right) \times\left(t_{0}-4 K r, t_{0}\right] .
\end{aligned}
$$

Lemma 3.3. Given $K>\sigma>\beta>0, \beta<\frac{1}{2}, Q_{i}(i=1, \cdots, 4)$ is defined as in (3-13). Let $u \geq 0$ be a solution of (1-1) in $Q_{4}$. If $\inf _{Q_{1}} u \geq 1$, then $\inf _{Q_{2}} u>\frac{1}{L}$, where $L>0$ depends only on $n, \sigma, K$ and the structure conditions. 
Proof. We first give some preliminaries. Let $T$ be an affine transformation that normalizes $S_{\phi}\left(x_{0}, r_{0}\right)=S_{\phi}\left(x_{0}, 4 r\right)$, and $T_{p}$ be an affine transformation defined by

$$
T_{p}:(x, t) \rightarrow\left(T x, \frac{t-\left(t_{0}-4 K r\right)}{4 r}\right) .
$$

The images of $\left\{Q_{i}\right\}_{i=1}^{4}$ under $T_{p}$ are denoted by

$$
\begin{aligned}
& Q_{4}^{*}=S_{4}^{*} \times(0, K], \quad \text { with } S_{4}^{*} \text { normalized, } \\
& Q_{3}^{*}=S_{3}^{*} \times(\beta, K], \\
& Q_{2}^{*}=S_{2}^{*} \times(\sigma, K], \\
& Q_{1}^{*}=S_{1}^{*} \times(0, K] .
\end{aligned}
$$

As before, $u^{*}(z)=u\left(T_{p}^{-1} z\right)$ satisfies $(3-5)$ in $Q_{4}^{*}$ with $\psi_{r_{0}}$ instead of $\psi_{r}$. Without loss of generality, we may assume that $u>0$. For $0<\varepsilon<1$, we claim that $u^{* \varepsilon}=\left(u^{*}\right)^{\varepsilon}$ satisfies the following:

$$
L_{\psi_{r_{0}}}\left(u^{* \varepsilon}\right) \geq \frac{(1-\varepsilon)}{\varepsilon u^{* \varepsilon}} \frac{\left|D\left(u^{* \varepsilon}\right)\right|^{2}}{\Delta \phi^{*}} .
$$

Obviously, $L_{\psi_{r_{0}}}=L_{\phi^{*}}$, where $\phi^{*}$ is defined as in (3-8) with $\psi_{r_{0}}$ instead of $\psi_{r}$. It is easy to check that

$$
\begin{aligned}
L_{\psi_{r_{0}}}\left(u^{* \varepsilon}\right) & =\varepsilon u^{*(\varepsilon-1)} u_{t}^{*}-\operatorname{tr}\left(\left(D^{2} \phi^{*}\right)^{-1} D\left(\varepsilon u^{*(\varepsilon-1)} D u\right)\right) \\
& =\varepsilon u^{*(\varepsilon-1)} u_{t}^{*}-\operatorname{tr}\left(\left(D^{2} \phi^{*}\right)^{-1}\left(\varepsilon u^{*(\varepsilon-1)} D^{2} u^{*}+\varepsilon(\varepsilon-1) u^{*(\varepsilon-2)} D u^{*}\left(D u^{*}\right)^{\prime}\right)\right) \\
& =\varepsilon(1-\varepsilon) u^{*(\varepsilon-2)}\left\langle\left(D^{2} \phi^{*}\right)^{-1} D u^{*}, D u^{*}\right\rangle \\
& \geq \varepsilon(1-\varepsilon) u^{*(\varepsilon-2)} \frac{\left|D u^{*}\right|^{2}}{\Delta \phi^{*}},
\end{aligned}
$$

where we have used the inequality

$$
\left\langle\left(D^{2} \phi^{*}\right)^{-1} D u, D u\right\rangle \geq \frac{|D u|^{2}}{\Delta \phi^{*}}
$$

for the proof of which, see Lemma 2.1 in [Ca-Gu2].

Let us recall some properties of normalization $\phi^{*}$ of $\phi$ in $S\left(x_{0}, r_{0}\right)$ (see [Ca-Gu2]). $\phi^{*}$ is a strictly convex function with $\phi^{*}=0$ on $\partial S_{4}^{*}$ and $-1 \leq \phi^{*} \leq 0$ in $S_{4}^{*}$. $\left|D \phi^{*}\right| \leq C_{\beta, n}$ on $S_{3}^{*}$ and $\left|D \phi^{*}\right| \geq C_{n}$ for $x \notin S_{1}^{*}$. Let $H_{\eta}=\left\{x \in S_{3}^{*}: \Delta \phi^{*}>\frac{1}{\eta}\right\}$ for $\eta>0$. An argument in [Ca-Gu2] gives

$$
\left|H_{\eta}\right| \leq \eta \int_{S_{3}^{*}} \Delta \phi^{*} d x \leq C_{\beta, n} \eta
$$

Let $\widetilde{H_{\eta}}$ be an open subset in $S_{4}^{*}$ such that $\overline{H_{\eta}} \subset \widetilde{H_{\eta}}$ and $\left|\widetilde{H_{\eta}} \backslash H_{\eta}\right| \leq \eta$. Take $f_{\eta}(x)$ to be a cuf-off function such that $f_{\eta} \in C_{0}^{\infty}\left(\widetilde{H_{\eta}}\right), 0 \leq f_{\eta} \leq 1$, and $f_{\eta}=1$ on $H_{\eta}$.

Let $h(z)=\frac{-\phi^{*}(x) t}{4 K}$. Then $0 \leq h \leq \frac{1}{4}$ on $Q_{4}^{*}$. We shall construct an auxiliary function. To this end, consider the boundary value problem for parabolic MongeAmpère equation:

$$
\begin{cases}-w_{t} \operatorname{det} D^{2} w=F(x, t), & \text { in } Q_{4}^{*} \\ w=\eta_{1}\left(\eta_{2}|x|^{2}-t\right), & \text { on } \partial_{p} Q_{4}^{*}\end{cases}
$$


where $F=(n+1)^{-(n+1)} \operatorname{det} D^{2} \phi^{*}\left(f_{\eta} L_{\phi^{*}}(h)+\eta_{0}\right)^{n+1}>0,0<\eta_{0}, \eta_{1}<1,0<$ $\eta_{2}<\beta$, and $L_{\phi^{*}}(h)=\frac{1}{4 K}\left(-\phi^{*}(x)+n t\right)$. The existence of classical solution $w \in$ $C^{2,1}\left(Q_{4}^{*}\right) \cap C\left(\overline{Q_{4}^{*}}\right)$, which is parabolically convex, i.e., convex in $x$ and nonincreasing in $t$, can be found in [W-W].

Now define the following auxiliary function:

$$
v(z)=u^{* \varepsilon}(z)-\left(h(z)+w(z)+\eta_{0} t\right), \quad \text { for } z=(x, t) .
$$

Since $h$ is strictly positive on $Q_{2}^{*}$, we will show this lemma by showing that $w$ is small and

$$
\delta=\min \left\{v(z): z \in \overline{Q_{4}^{*}}\right\}=v(P)=v\left(x^{\prime}, t^{\prime}\right)
$$

is positive or close to zero. It is convenient to distinguish the following three cases:

Case 1. $P \in Q_{1}^{*}$.

Case 2. $P \in \overline{Q_{4}^{*}} \backslash Q_{3}^{*}$.

Case 3. $P \in Q_{3}^{*} \backslash Q_{1}^{*}$.

Case 1. Suppose $P \in Q_{1}^{*}$. We first show that the correction function $w$ is very small if $\eta, \eta_{0}$ and $\eta_{1}$ are sufficiently small. Since $w$ is nonincreasing in $t$,

$$
\sup _{Q_{4}^{*}} w \leq \eta_{1} \eta_{2} \sup _{x \in S_{4}^{*}}|x|^{2} \leq \eta_{1}
$$

On the other hand, by Aleksandrov-Bakelman-Pucci-Krylov-Tso's estimate ([T]), we have

$$
\begin{aligned}
\sup _{Q_{4}^{*}}(-w) & \leq \sup _{\partial_{p} Q_{4}^{*}}(-w)+C\left\{\operatorname{diam}\left(S_{4}^{*}\right)^{n} \iint_{Q_{4}^{*}} F d x d t\right\}^{1 /(n+1)} \\
& \leq \eta_{1} K+C\left\{\int_{0}^{K} \int_{S_{4}^{*}}\left(f_{\eta} L_{\phi^{*}}(h)+\eta_{0}\right)^{n+1} \operatorname{det} D^{2} \phi^{*} d x d t\right\}^{1 /(n+1)} \\
& \leq \eta_{1} K+C\left\{\int_{\widetilde{H_{\eta}}} \operatorname{det} D^{2} \phi^{*} d x+\eta_{0}^{n+1} \mu^{*}\left(S_{4}^{*}\right)\right\}^{1 /(n+1)} .
\end{aligned}
$$

Recalling that $\mu^{*}\left(S_{4}^{*}\right) \leq C_{n}$ and noting that (1-3) is affine invariant, i.e.,

$$
\frac{\left|\widetilde{H_{\eta}}\right|}{\left|S_{4}^{*}\right|}<\delta_{2} \quad \text { implies } \quad \frac{\mu^{*}\left(\widetilde{H_{\eta}}\right)}{\mu^{*}\left(S_{4}^{*}\right)}<\delta_{1},
$$

we have that $\sup _{Q_{4}^{*}}|w| \leq \eta^{*}$, for arbitrary $\eta^{*}$, if $\eta, \eta_{0}$ and $\eta_{1}$ are sufficiently small.

We can now estimate $\delta$. Obviously if $\eta_{0} \leq \frac{1}{8 K}$ and $\eta^{*} \leq \frac{1}{8}$

$$
\delta \geq 1-\frac{1}{4}-\eta^{*}-\eta_{0} K \geq \frac{1}{2}
$$

Therefore, for $z \in Q_{2}^{*}$

$$
\begin{aligned}
u^{* \varepsilon}(z) & =v(z)+\left(h(z)+w(z)+\eta_{0} t\right) \\
& \geq \frac{1}{2}+0-\eta^{*}+0,
\end{aligned}
$$

which implies $u^{*}(z) \geq\left(\frac{1}{4}\right)^{1 / \varepsilon}$, for $z \in Q_{2}^{*}$.

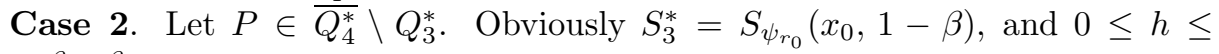
$\max \left(\frac{\beta}{4}, \frac{\beta}{4 K}\right)$ on $Q_{4}^{*} \backslash Q_{3}^{*}$ by the definition of $Q_{3}^{*}$. Then we have

$$
\delta \geq 0-C \beta-\eta^{*}-\eta_{0} K .
$$


It follows that for $z \in Q_{2}^{*}$

$$
\begin{aligned}
u^{* \varepsilon}(z) & =v(z)+\left(h(z)+w(z)+\eta_{0} t\right) \\
& \geq-C \beta-\eta^{*}-\eta_{0} K+\frac{\frac{1}{2} \sigma}{4 K}-\eta^{*}+0 \\
& \geq \frac{\sigma}{16 K} .
\end{aligned}
$$

Here we choose $\beta, \eta_{0}$, and $\eta^{*}$ sufficiently small depending on $C, \sigma$, and $K$. Thus, $u^{*}(z) \geq\left(\frac{\sigma}{16 K}\right)^{1 / \varepsilon}$ on $Q_{2}^{*}$.

Case 3. Let $P \in Q_{3}^{*} \backslash Q_{1}^{*}$. Since $P$ is a minimum point, we have that $D v(P)=0$, $D^{2} v(P) \geq 0$, and $v_{t}(P) \leq 0$. Therefore

$$
L_{\phi^{*}}(v)=v_{t}-\operatorname{tr}\left(\left(D^{2} \phi^{*}\right)^{-1} D^{2} v\right) \leq 0, \quad \text { at } P .
$$

It implies that

$$
L_{\phi^{*}}\left(u^{* \varepsilon}\right) \leq L_{\phi^{*}}(h+w)+\eta_{0}, \quad \text { at } P .
$$

Since $w$ is a parabolically convex solution of (BVP), we have

$$
\begin{aligned}
F & =\left(-w_{t}\right) \operatorname{det} D^{2} w \\
& \leq\left[\frac{-w_{t}+\operatorname{tr}\left(\left(D^{2} \phi^{*}\right)^{-1} D^{2} w\right)}{n+1}\right]^{n+1} \operatorname{det} D^{2} \phi^{*},
\end{aligned}
$$

i.e.,

$$
\begin{aligned}
& (n+1)^{-(n+1)} \operatorname{det} D^{2} \phi^{*}\left(f_{\eta} L_{\phi^{*}}(h)+\eta_{0}\right)^{n+1} \\
\leq & (n+1)^{-(n+1)}\left(-L_{\phi^{*}}(w)\right)^{n+1} \operatorname{det} D^{2} \phi^{*}
\end{aligned}
$$

It proceeds to

$$
f_{\eta} L_{\phi^{*}}(h)+\eta_{0} \leq-L_{\phi^{*}}(w)
$$

From (3-14), (3-15), it follows that at $P$

$$
\frac{(1-\varepsilon)}{\varepsilon u^{* \varepsilon}} \frac{\left|D\left(u^{* \varepsilon}\right)\right|^{2}}{\Delta \phi^{*}} \leq L_{\phi^{*}}(h)-\left(f_{\eta} L_{\phi^{*}}(h)+\eta_{0}\right)+\eta_{0}
$$

Therefore

$$
\frac{(1-\varepsilon)}{\varepsilon u^{* \varepsilon}} \frac{\left|D\left(u^{* \varepsilon}\right)\right|^{2}}{\Delta \phi^{*}} \leq\left(1-f_{\eta}\right) L_{\phi^{*}}(h), \quad \text { at } P .
$$

Since $\eta_{2}<\beta, w(x, t) \leq 0$ for $x \in \partial S_{4}^{*}$ and $t \geq \beta$. By the property of convex functions (see $[\mathrm{Ca}-\mathrm{Gu} 2]$ ), we have that for $x \in S_{3}^{*}$

$$
|D w(x, t)| \leq \frac{-w(x, t)}{d\left(S_{3}^{*}, \partial S_{4}^{*}\right)} .
$$

Therefore, we obtain at $P$

$$
\begin{aligned}
\left|D\left(u^{* \varepsilon}\right)\right| & =|D h+D w| \\
& \geq|D h|-|D w| \\
& \geq C \beta-\frac{\sup |w|}{d\left(S_{3}^{*}, \partial S_{4}^{*}\right)} .
\end{aligned}
$$


Obviously, if $\beta$ is fixed, taking $\eta$ and $\eta_{0}$ sufficiently small, then $\sup |w| \leq \eta^{*}$ is also small enough, and therefore we have

$$
\left|D\left(u^{* \varepsilon}\right)\right|(P) \geq C .
$$

By $(3-16)\left(P=\left(x^{\prime}, t^{\prime}\right)\right), L_{\phi^{*}}(h)=\left(-\phi^{*}(x)+n t\right) / 4 K$, we obtain

$$
C \frac{1-\varepsilon}{\varepsilon} \frac{1}{u^{* \varepsilon}(P)} \leq\left(1-f_{\eta}\left(x^{\prime}\right)\right) \Delta \phi^{*}\left(x^{\prime}\right) .
$$

Since the LHS of above inequality is positive, $f_{\eta}\left(x^{\prime}\right) \neq 1$. This implies that $x^{\prime} \notin H_{\eta}$ and $\Delta \phi^{*}\left(x^{\prime}\right) \leq \frac{1}{\eta}$. Therefore

$$
u^{* \varepsilon}(P) \geq \frac{C \eta(1-\varepsilon)}{\varepsilon} .
$$

Obviously, if we choose $\varepsilon$ sufficiently small, then

$$
\delta \geq \frac{C \eta(1-\varepsilon)}{\varepsilon}-\frac{1}{4}-\eta^{*}-\eta_{0} K \geq 1
$$

It follows that for $z \in Q_{2}^{*}$

$$
\begin{aligned}
u^{* \varepsilon}(z) & \geq \delta+\left(h(z)+w(z)+\eta_{0} t\right) \\
& \geq 1-\eta^{*} \\
& \geq \frac{1}{2}
\end{aligned}
$$

Therefore, $u(z) \geq\left(\frac{1}{2}\right)^{1 / \varepsilon}$. This completes the proof of Lemma 3.3.

Repeatedly applying Lemma 3.1-3.3, we obtain

Theorem 3.1. Let $z_{0}=\left(x_{0}, t_{0}\right)$ and $z_{0}^{\prime}=\left(x_{0}, t_{0}+2 r\right)$. Let $u \geq 0$ be a solution of (1-1) in $S\left(x_{0}, 4 r\right) \times\left(t_{0}-r, t_{0}+2 r\right]$. Assume that

$$
\inf \left\{u(z): z \in Q\left(z_{0}^{\prime}, 2 r\right)\right\} \leq 1
$$

Then there exists $M_{1}$ such that

$$
\mathcal{M}\left(\left\{z \in Q\left(z_{0}, r\right): u(z)<M_{1}\right\}\right)>\varepsilon_{0} \mathcal{M}\left(Q\left(z_{0}, r\right)\right),
$$

where $\varepsilon_{0}$ is the constant in Lemma 3.1 and $M_{1}$ depends only on $n$ and the structure conditions.

Proof. By contradiction. If (3-17) is false, applying Lemma 3.1 to $M_{0} u / M_{1}$, we obtain

$$
\inf \left\{u(z): z \in Q\left(z_{0}, \frac{r}{2}\right)\right\}>\frac{M_{1}}{M_{0}} .
$$

Therefore, by applying Lemma 3.2 to $M_{0}^{2} u / M_{1}$, it follows that

$$
\inf \left\{u(z): z \in S\left(x_{0}, \frac{r}{4}\right) \times\left(t_{0}, t_{0}+\tau \frac{r}{2}\right]\right\}>\frac{M_{1}}{M_{0}^{2}} .
$$

By (3-18) and (3-19)

$$
\inf \left\{u(z): z \in S\left(x_{0}, \frac{r}{4}\right) \times\left(t_{0}-\frac{r}{2}, t_{0}+\tau \frac{r}{2}\right]\right\}>\frac{M_{1}}{M_{0}^{2}} .
$$


By applying Lemma 3.3 with $r / 4$ instead of $r, K=(1+\tau) / 2$ and $\sigma=\tau / 2$ to the solution $M_{0}^{2} u / M_{1}$, we obtain

$$
\inf \left\{u(z): z \in S\left(x_{0}, \frac{r}{2}\right) \times\left(t_{0}+(\tau-1) \frac{r}{2}, t_{0}+\tau \frac{r}{2}\right]\right\}>\frac{M_{1}}{M_{0}^{2} L} .
$$

By applying Lemma 3.2 and Lemma 3.3 once again, we find that

$$
\inf \left\{u(z): z \in S\left(x_{0}, \frac{r}{2}\right) \times\left(t_{0}+(2 \tau-1) \frac{r}{2}, t_{0}+2 \tau \frac{r}{2}\right]\right\}>\frac{M_{1}}{M_{0}}\left(\frac{1}{M_{0} L}\right)^{2} .
$$

Continuing with this process, we conclude that at $k$ th-step

$$
\inf \left\{u(z): z \in S\left(x_{0}, \frac{r}{2}\right) \times\left(t_{0}+(k \tau-1) \frac{r}{2}, t_{0}+k \tau \frac{r}{2}\right]\right\}>\frac{M_{1}}{M_{0}} \frac{1}{\left(M_{0} L\right)^{k}} .
$$

Hence, by choosing $k$ such that $k \geq 4 / \tau>k-1$, it follows that

$$
\inf \left\{u(z): z \in S\left(x_{0}, \frac{r}{2}\right) \times\left(t_{0}-\frac{r}{2}, t_{0}+2 r\right]\right\}>\frac{M_{1}}{M_{0}} \frac{1}{\left(M_{0} L\right)^{k}} .
$$

We now apply Lemma 3.3 with $\frac{r}{2}$ instead of $r, K=5 / 4, \sigma=1 / 8$ to the solution $\frac{M_{0}}{M_{1}}\left(M_{0} L\right)^{k} u$, and then there exists $L_{1}$ such that

$$
\inf \left\{u(z): z \in S\left(x_{0}, r\right) \times\left(t_{0}-\frac{r}{4}, t_{0}+2 r\right]\right\}>\frac{M_{1}}{M_{0}\left(M_{0} L\right)^{k} L_{1}} .
$$

Once again applying Lemma 3.3 with $K=\frac{9}{16}$ and $\sigma=\frac{1}{16}$ to the solution $\frac{M_{0}\left(M_{0} L\right)^{k} L_{1}}{M_{1}} u$, we obtain that there exists $L_{2}$ such that

$$
\inf \left\{u(z): z \in S\left(x_{0}, 2 r\right) \times\left(t_{0}, t_{0}+2 r\right]\right\}>\frac{M_{1}}{M_{0}\left(M_{0} L\right)^{k} L_{1} L_{2}} .
$$

If we choose $M_{1}>M_{0}\left(M_{0} L\right)^{k} L_{1} L_{2}$, then the inequality above contradicts the assumption of Theorem 3.1. Thus, we finish the proof.

To end this section, we give another theorem which will be used in discussing the power decay of distribution function of solutions of (1-1).

Theorem 3.2. Let $z_{0}=\left(x_{0}, t_{0}\right)$ and $K>\sigma>0$. Let $u \geq 0$ be a solution of (1-1) in $S\left(x_{0}, 2^{i+1} r\right) \times\left(t_{0}-K r, t_{0}\right]$. Assume that

$$
\inf \left\{u(z): z \in S\left(x_{0}, r\right) \times\left(t_{0}-K r, t_{0}\right]\right\} \geq 1 .
$$

Then there exists $L_{0}$ depending on $K, \sigma, i, n$ and the structure conditions such that

$$
\inf \left\{u(z): z \in S\left(x_{0}, 2^{i} r\right) \times\left(t_{0}-(K-\sigma) r, t_{0}\right]\right\}>\frac{1}{L_{0}} .
$$

Proof. To show this theorem, we will repeatedly apply Lemma 3.3. For $1 \leq j \leq i$, let $t_{j}=t_{0}-K r+\frac{j}{i} \sigma r$. By Lemma 3.3 with $\frac{\sigma}{4 i}, K / 4$ instead of $\sigma, K$, respectively, we obtain that there exists $L_{1}$ such that

$$
\inf \left\{u(z): z \in S\left(x_{0}, 2 r\right) \times\left(t_{1}, t_{0}\right]\right\}>\frac{1}{L_{1}} .
$$


Then apply Lemma 3.3 with $2 r,\left(K-\frac{\sigma}{i}\right) / 8, \sigma / 8 i$ instead of $r, K, \sigma$, respectively, to the function $L_{1} u$, and then there exists $L_{2}$ such that

$$
\inf \left\{u(z): z \in S\left(x_{0}, 2^{2} r\right) \times\left(t_{2}, t_{0}\right]\right\}>\frac{1}{L_{1} L_{2}} .
$$

Continue this process and there exist constants $L_{3}, \ldots, L_{i}$ such that

$$
\inf \left\{u(z): z \in S\left(x_{0}, 2^{i} r\right) \times\left(t_{i}, t_{0}\right]\right\}>\frac{1}{L_{1} \cdots L_{i}} .
$$

The theorem follows immediately.

\section{The Harnack inequality}

The purpose of this section is to derive the power decay of distribution functions, the weak Harnack inequality, and the Harnack inequality for nonnegative solutions of (1-1), by using the estimates established in $\S 3$ and the Calderón-Zygmund decomposition (Theorem 2.2).

Given $z_{0}=\left(x_{0}, t_{0}\right)$ and $R>0$, let

$$
\begin{aligned}
& Q^{+}=S\left(x_{0}, R\right) \times\left(t_{0}+R, t_{0}+2 R\right], \\
& Q=S\left(x_{0}, 2 R\right) \times\left(t_{0}-\frac{3 R}{2}, t_{0}+\frac{R}{2}\right], \\
& Q^{-}=S\left(x_{0}, R\right) \times\left(t_{0}-R, t_{0}\right], \\
& Q_{B}=S\left(x_{0}, 8 \theta^{2} R\right) \times\left(t_{0}-\frac{3 R}{2}, t_{0}+2 R\right],
\end{aligned}
$$

where $\theta$ is the engulfing constant in property $(\mathrm{C})$ in $\S 2$. For $z^{\prime}=\left(x^{\prime}, t^{\prime}\right)$ we use the following notations:

$$
\begin{aligned}
& Q\left(z^{\prime}, \rho\right)=S\left(x^{\prime}, \rho\right) \times\left(t^{\prime}-\rho, t^{\prime}\right], \\
& \widetilde{Q}\left(z^{\prime}, \rho\right)=S\left(x^{\prime}, \rho\right) \times\left(t^{\prime}-\frac{\rho}{2}, t^{\prime}+\frac{\rho}{2}\right] .
\end{aligned}
$$

We first show the following lemma.

Lemma 4.1. Let $u \geq 0$ be a solution of (1-1) in $Q_{B}$. Assume that $\inf _{Q^{+}} u \leq 1$. Let $z^{\prime}=\left(x^{\prime}, t^{\prime}\right) \in Q$ and suppose

$$
\mathcal{M}\left(\left\{z \in \widetilde{Q}\left(z^{\prime}, \rho\right): u(z)>M\right\}\right) \geq \lambda \mathcal{M}\left(\widetilde{Q}\left(z^{\prime}, \rho\right)\right),
$$

where $\lambda=1-\varepsilon_{0}$ and $\varepsilon_{0}$ is the constant in Lemma 3.1. We conclude that

(i) if $\rho<\frac{R}{6}$, then $\rho \leq C_{2} M^{-\delta_{0}} R$,

(ii) If $M>M_{2}$, then $\rho<\frac{R}{6}$,

where $C_{2}, \delta_{0}, M_{2}>0$ depend only on $n$ and the structure conditions.

Proof. We first prove (i). Suppose that $\rho<R / 6$. Let $t_{0}^{\prime}=t^{\prime}+\rho / 2, t_{i+1}^{\prime}=t_{i}^{\prime}+2^{i} \rho$ and $z_{i}^{\prime}=\left(x^{\prime}, t_{i}^{\prime}\right)$ for $i \geq 0$. Let $j$ be the integer such that $t_{j}^{\prime}<t_{0}+\frac{3 R}{4} \leq t_{j+1}^{\prime}$. Obviously

$$
t_{1}^{\prime}=t^{\prime}+\frac{3 \rho}{2}<t_{0}+\frac{R}{2}+\frac{3}{2} \cdot \frac{R}{6}=t_{0}+\frac{3 R}{4},
$$

so $j \geq 1$. By the definition of $j$

$$
t_{j}^{\prime}=t^{\prime}+\left(2^{j}-\frac{1}{2}\right) \rho<t_{0}+\frac{3 R}{4} \leq t^{\prime}+\left(2^{j+1}-\frac{1}{2}\right) \rho=t_{j+1}^{\prime} .
$$


It follows that

$$
t_{0}-\frac{3 R}{2}+\left(2^{j}-\frac{1}{2}\right) \rho<t_{0}+\frac{3 R}{4} \leq t_{0}+\frac{R}{2}+\left(2^{j+1}-\frac{1}{2}\right) \rho,
$$

and hence

$$
\frac{R}{8} \leq 2^{j} \rho<3 R
$$

Clearly

$$
\begin{aligned}
t_{j+4}^{\prime} & =t_{j+1}^{\prime}+\left(2^{j+4}-2^{j+1}\right) \rho \\
& \geq t_{0}+\frac{3 R}{4}+14 \cdot \frac{R}{8} \\
& >t_{0}+2 R .
\end{aligned}
$$

Since $\widetilde{Q}\left(z^{\prime}, \rho\right)=Q\left(z_{0}^{\prime}, \rho\right)$, by Lemma 3.1 and $(4-1)$

$$
\inf \left\{u(z): z \in Q\left(z_{0}^{\prime}, \frac{\rho}{2}\right)\right\}>\frac{M}{M_{0}} .
$$

Repeatedly applying Theorem 3.1, we obtain for $i \geq 1$

$$
\inf \left\{u(z): z \in Q\left(z_{i}^{\prime}, 2^{i-1} \rho\right)\right\}>\frac{M}{M_{0} M_{1}^{i}} .
$$

By (4-2) and (4-3)

$$
\inf \left\{u(z): z \in S\left(x^{\prime}, 2^{j} \rho\right) \times\left(t_{0}+\frac{3 R}{4}, t_{0}+2 R\right]\right\}>\frac{M}{M_{0} M_{1}^{j+4}} .
$$

Noting that $2^{j} \rho \geq R / 8$, applying Theorem 3.2 with $r=R / 8, K=10, \sigma=2$ and $i$ satisfying $2^{i} \geq 16 \theta$, where $\theta$ is the engulfing constant, to the solution $M_{0} M_{1}^{j+4} u / M$, we obtain

$$
\inf \left\{u(z): z \in S\left(x^{\prime}, 2^{i} \frac{R}{8}\right) \times\left(t_{0}+R, t_{0}+2 R\right]\right\}>\frac{M}{M_{0} M_{1}^{j+4} L_{0}} .
$$

By the engulfing property

$$
S\left(x^{\prime}, 2^{i-3} R\right) \supset S\left(x^{\prime}, 2 \theta R\right) \supset S\left(x_{0}, 2 R\right),
$$

then from (4-4) and $\inf _{Q^{+}} u \leq 1$, we conclude that $M<M_{0} M_{1}^{j+4} L_{0}$. Therefore

$$
j>\log _{2} \frac{M}{M_{0} M_{1}^{4} L_{0}} / \log _{2} M_{1} .
$$

We have

$$
\begin{aligned}
\rho & <2^{-j} \cdot 3 R \\
& <2^{-\log _{2} \frac{M}{M_{0} M_{1}^{4} L_{0}} / \log _{2} M_{1}} \cdot 3 R \\
& =3 \cdot 2^{\frac{\log _{2}\left(M_{0} M_{1}^{4} L_{0}\right)}{\log _{2} M_{1}}} \cdot M^{-\frac{1}{\log _{2} M_{1}}} \cdot R .
\end{aligned}
$$

This finishes the proof of (i). 
Now turn to show (ii). Suppose that $\rho \geq R / 6$. We want to deduce a contradiction if $M_{2}$ is sufficiently large. Obviously

$$
\begin{aligned}
t_{5}^{\prime} & =t^{\prime}+\left(2^{5}-\frac{1}{2}\right) \rho \\
& \geq t_{0}-\frac{3 R}{2}+\frac{63}{2} \cdot \frac{R}{6} \\
& >t_{0}+2 R .
\end{aligned}
$$

As before, by Lemma 3.1 and (4-1)

$$
\inf \left\{u(z): z \in Q\left(z_{0}^{\prime}, \frac{\rho}{2}\right)\right\}>\frac{M}{M_{0}} .
$$

Since $\frac{\rho}{2}>\frac{R}{12}$, by $(4-3)$ we have

$$
\inf \left\{u(z): z \in S\left(x^{\prime}, \frac{R}{12}\right) \times\left(t_{0}+\frac{R}{2}, t_{0}+2 R\right]\right\}>\frac{M}{M_{0} M_{1}^{5}} .
$$

Applying Theorem 3.2 with $r=R / 12, K=18, \sigma=6$, to $M_{0} M_{1}^{5} u / M$, we obtain

$$
\inf \left\{u(z): z \in S\left(x^{\prime}, 2^{i} \frac{R}{12}\right) \times\left(t_{0}+R, t_{0}+2 R\right]\right\}>\frac{M}{M_{0} M_{1}^{5} L_{0}},
$$

where $i$ is taken such that $2^{i} \geq 24 \theta$ and $\theta$ is the engulfing constant. Since

$$
S\left(x^{\prime}, 2^{i} \frac{R}{12}\right) \supset S\left(x^{\prime}, 2 \theta R\right) \supset S\left(x_{0}, 2 R\right),
$$

we have

$$
\inf _{Q^{+}} u>\frac{M}{M_{0} M_{1}^{5} L_{0}},
$$

which contradicts with $\inf _{Q^{+}} u \leq 1$ if $M>M_{0} M_{1}^{5} L_{0}=M_{2}$. We conclude (ii).

We are now in a position to show the power decay of the distribution function of $u$.

Theorem 4.1. Let $u \geq 0$ satisfy (1-1) in $Q_{B}$. Assume that $\inf _{Q^{+}} u \leq 1$. Then for $i \geq 1$

$$
\mathcal{M}\left(\left\{z \in Q^{-}: u(z)>\bar{K} M^{i}\right\}\right) \leq \gamma^{i} \mathcal{M}(Q),
$$

where $0<\gamma<1, \bar{K}, M$ are depending only on $n$ and the structure conditions.

Proof. Set $\bar{t}=t_{0}-R / 2$ and $\bar{z}=\left(x_{0}, \bar{t}\right)$. Then $Q=\widetilde{Q}(\bar{z}, 2 R)$ and $Q^{-}=\widetilde{Q}(\bar{z}, R)$. Let $\tau_{1}=2$ and for $i \geq 2$

$$
\tau_{i}=2\left(1-\sum_{j=2}^{i}\left[C_{2}\left(\bar{K} M^{j}\right)^{-\delta_{0}}\right]^{1 / p}\right),
$$

where $C_{2}$ and $\delta_{0}$ are the constants in Lemma $4.1 ; p$ is the constant in Property (D) in $\S 2 ; \bar{K}$ and $M$ (large numbers) will be chosen later. Note that $1 \leq \tau_{i} \leq 2$ if $\bar{K}$ and $M>1$ are sufficiently large. To prove (4-5), it is sufficient to show that for $i \geq 1$

$$
\mathcal{M}\left(\left\{z \in \widetilde{Q}\left(\bar{z}, \tau_{i} R\right): u(z)>\bar{K} M^{i}\right\}\right) \leq \gamma^{i} \mathcal{M}(Q)
$$


Now begin to choose $\gamma$ in (4-6). For $\varepsilon_{0}$ the constant in Theorem 3.1, $\lambda=1-\varepsilon_{0}$, let $\gamma_{0}=c(\lambda)<1$ be the constant in Theorem 2.2. Choose $m$ and $\gamma$ such that

$$
\gamma_{1}=\frac{m+1}{m} \gamma_{0}<\gamma<1, \quad \text { and } \quad \lambda<\gamma .
$$

Now fix $m$ and $\gamma$. We want to show (4-6) by induction.

Case $i=1$. Since $\inf _{Q^{+}} u \leq 1$, by Theorem 3.1 and Lemma 3.1, we have

$$
\mathcal{M}\left(\left\{z \in \widetilde{Q}(\bar{z}, 2 R): u(z)>M_{1}\right\}\right) \leq \lambda \mathcal{M}(\widetilde{Q}(\bar{z}, 2 R)) .
$$

Take $\bar{K} \geq 1$ and $M \geq M_{1}$. Then (4-6) holds when $i=1$.

Case $i=2$. Set

$$
E_{2}=\left\{z \in \widetilde{Q}\left(\bar{z}, \tau_{2} R\right): u(z)>\bar{K} M^{2}\right\} .
$$

We only need to consider open sections in the measure argument. By the CalderónZygmund decomposition (Theorem 2.2) at the level $\lambda$ of $E_{2}$, there exists $\left\{\widetilde{Q}\left(z_{k}, \rho_{k}\right)\right\}$ such that

$$
\mathcal{M}\left(E_{2}\right) \leq \gamma_{1} \mathcal{M}\left(\bigcup_{k} \widetilde{Q}^{m}\left(z_{k}, \rho_{k}\right)\right)
$$

and

$$
\frac{\mathcal{M}\left(\widetilde{Q}\left(z_{k}, \rho_{k}\right) \cap E_{2}\right)}{\mathcal{M}\left(\widetilde{Q}\left(z_{k}, \rho_{k}\right)\right)}=\lambda
$$

From (4-8) and by applying Theorem $3.1 \mathrm{~m}$ times, we have

$$
u>\frac{\bar{K} M^{2}}{M_{1}^{m}}, \quad \text { on } \Gamma^{m}=\bigcup_{k} \widetilde{Q}^{m}\left(z_{k}, \rho_{k}\right) .
$$

By Lemma 4.1, if $M \geq M_{2}$, then

$$
\rho_{k} \leq C_{2}\left(\bar{K} M^{2}\right)^{-\delta_{0}} 2 R .
$$

Since $z_{k} \in E_{2}$, we have $\widetilde{Q}\left(z_{k}, \rho_{k}\right) \subset \widetilde{Q}(\bar{z}, 2 R)$ by the definition of $\tau_{2}$ and the following variant of Property (D) in $\S 2$ : for $0<s_{2}<s_{1} \leq 1$

$$
\widetilde{Q}\left(z,\left(s_{1}-s_{2}\right)^{p} R\right) \subset \widetilde{Q}\left(z_{0}, s_{1} R\right), \quad \text { for } z \in \widetilde{Q}\left(z_{0}, s_{2} R\right) .
$$

Let

$$
B=\left\{z \in \widetilde{Q}(\bar{z}, 2 R)=Q: u>\frac{\bar{K} M^{2}}{M_{1}^{m}}\right\} .
$$

Take $M>M_{1}^{m}$. If $\mathcal{M}\left(E_{2}\right) \leq \gamma \mathcal{M}(B)$, then by the conclusion of case 1

$$
\begin{aligned}
\mathcal{M}\left(E_{2}\right) & \leq \gamma \mathcal{M}(\{z \in \widetilde{Q}(\bar{z}, 2 R): u>\bar{K} M\}) \\
& \leq \gamma^{2} \mathcal{M}(Q),
\end{aligned}
$$


which is (4-6) for $i=2$. If $\mathcal{M}\left(E_{2}\right)>\gamma \mathcal{M}(B)$, let us assume by contradiction that $\mathcal{M}\left(E_{2}\right)>\gamma^{2} \mathcal{M}(Q)$. Obviously by (4-9) and (4-7)

$$
\begin{aligned}
\mathcal{M}\left(\Gamma^{m} \cap Q^{c}\right) & =\mathcal{M}\left(\Gamma^{m}\right)-\mathcal{M}\left(\Gamma^{m} \cap Q\right) \\
& \geq \frac{1}{\gamma_{1}} \mathcal{M}\left(E_{2}\right)-\mathcal{M}(B) \\
& \geq \frac{1}{\gamma_{1}} \mathcal{M}\left(E_{2}\right)-\frac{1}{\gamma} \mathcal{M}\left(E_{2}\right) \\
& \geq\left(\frac{1}{\gamma_{1}}-\frac{1}{\gamma}\right) \gamma^{2} \mathcal{M}(Q) .
\end{aligned}
$$

Let

$$
T=\sup \left\{t: \exists x \text { such that }(x, t) \in \Gamma^{m}\right\}
$$

Therefore

$$
\left(T-\left(t_{0}+\frac{R}{2}\right)\right) \cdot \mu\left(S\left(x_{0}, 2 R\right)\right) \geq\left(\frac{1}{\gamma_{1}}-\frac{1}{\gamma}\right) \gamma^{2} 2 R \mu\left(S\left(x_{0}, 2 R\right)\right) .
$$

This implies that there exists a point $z \in \Gamma^{m}$ above the hyperplane $t=t_{0}+R / 2+$ $C_{3} \gamma^{2} R$ with $C_{3}=2\left(\gamma_{1}^{-1}-\gamma^{-1}\right)$. Therefore, there exists a section $\widetilde{Q}\left(z_{k}, \rho_{k}\right)$ such that $z \in \widetilde{Q}^{m}\left(z_{k}, \rho_{k}\right)$ and this implies

$$
(m+1) \rho_{k} \geq\left(t_{0}+\frac{R}{2}+C_{3} \gamma^{2} R\right)-\left(t_{0}+\frac{R}{2}\right)=C_{3} \gamma^{2} R .
$$

By $(4-10)$

$$
C_{2}\left(\bar{K} M^{2}\right)^{-\delta_{0}} 2 R \geq \frac{C_{3}}{m+1} \gamma^{2} R,
$$

which gives a contradiction, if we choose $\bar{K}$ and $M$ such that

$$
\begin{aligned}
& 2 C_{2} \bar{K}^{-\delta_{0}}<\frac{C_{3}}{m+1}, \\
& M^{-\delta_{0}}<\gamma .
\end{aligned}
$$

Therefore we conclude (4-6) for $i=2$.

Suppose that (4-6) holds for $i$.

Case $i+1$. The argument in this case is similar to that in case $i=2$. We just give a sketch of the proof. Let

$$
E_{i+1}=\left\{z \in \widetilde{Q}\left(\bar{z}, \tau_{i+1} R\right): u(z)>\bar{K} M^{i+1}\right\} .
$$

By Theorem 2.2, there exists $\left\{\widetilde{Q}\left(z_{k}, \rho_{k}\right)\right\}$ such that

$$
\mathcal{M}\left(E_{i+1}\right) \leq \gamma_{1} \mathcal{M}\left(\Gamma_{1}^{m}\right),
$$

and

$$
\frac{\mathcal{M}\left(\widetilde{Q}\left(z_{k}, \rho_{k}\right) \cap E_{i+1}\right)}{\mathcal{M}\left(\widetilde{Q}\left(z_{k}, \rho_{k}\right)\right)}=\lambda,
$$

where $\Gamma_{1}^{m}=\bigcup_{k} \widetilde{Q}^{m}\left(z_{k}, \rho_{k}\right)$. By Lemma 4.1 and (4-13)

$$
\rho_{k} \leq C_{2}\left(\bar{K} M^{i+1}\right)^{-\delta_{0}} 2 R,
$$


which implies that $\widetilde{Q}\left(z_{k}, \rho_{k}\right) \subset \widetilde{Q}\left(\bar{z}, \tau_{i} R\right)$, since $z_{k} \in \widetilde{Q}\left(\bar{z}, \tau_{i+1} R\right)$. Obviously, by $(4-13)$,

$$
\Gamma_{1}^{m} \cap \widetilde{Q}\left(\bar{z}, \tau_{i} R\right) \subset B_{1}=\left\{z \in \widetilde{Q}\left(\bar{z}, \tau_{i} R\right): u>\frac{\bar{K} M^{i+1}}{M_{1}^{m}}\right\} .
$$

If $\mathcal{M}\left(E_{i+1}\right) \leq \gamma \mathcal{M}\left(B_{1}\right)$, then for $M>M_{1}^{m}$, by the induction assumption

$$
\begin{aligned}
\mathcal{M}\left(E_{i+1}\right) & \leq \gamma \mathcal{M}\left(\left\{z \in \widetilde{Q}\left(\bar{z}, \tau_{i} R\right): u>\bar{K} M^{i}\right\}\right) \\
& \leq \gamma^{i+1} \mathcal{M}(Q),
\end{aligned}
$$

which is (4-6) for case $\mathrm{i}+1$. If $\mathcal{M}\left(E_{i+1}\right)>\gamma \mathcal{M}\left(B_{1}\right)$, let us assume by contradiction that (4-6) is not valid for $i+1$. Then we have by (4-12)

$$
\begin{aligned}
\mathcal{M}\left(\Gamma_{1}^{m} \cap \widetilde{Q}^{c}\left(\bar{z}, \tau_{i} R\right)\right) & =\mathcal{M}\left(\Gamma_{1}^{m}\right)-\mathcal{M}\left(\Gamma_{1}^{m} \cap \widetilde{Q}\left(\bar{z}, \tau_{i} R\right)\right) \\
& \geq\left(\frac{1}{\gamma_{1}}-\frac{1}{\gamma}\right) \mathcal{M}\left(E_{i+1}\right) \\
& \geq\left(\frac{1}{\gamma_{1}}-\frac{1}{\gamma}\right) \gamma^{i+1} \mathcal{M}(Q) .
\end{aligned}
$$

This implies that there exists a point $z \in \Gamma_{1}^{m}$ above the hyperplane $t=\bar{t}+\tau_{i} R / 2+$ $C_{3} \gamma^{i+1} R$. Therefore, there exists $\widetilde{Q}\left(z_{k}, \rho_{k}\right)$ such that $z \in \widetilde{Q}^{m}\left(z_{k}, \rho_{k}\right)$ and hence

$$
(m+1) \rho_{k} \geq\left(\bar{t}+\frac{\tau_{i} R}{2}+C_{3} \gamma^{i+1} R\right)-\left(\bar{t}+\frac{\tau_{i} R}{2}\right)=C_{3} \gamma^{i+1} R,
$$

which contradicts with (4-14) if (4-11) holds. This proceeds immediately to the conclusion of the theorem.

By a standard argument, the weak Harnack inequality is an immediate corollary of Theorem 4.1 (see [Ca-C, p. 48]).

Corollary 4.1. Let $u \geq 0$ be a solution of (1-1) in $Q_{B}$. There exists $\delta_{3}>0$ such that

$$
\left(\frac{1}{\mathcal{M}\left(Q^{-}\right)} \iint_{Q^{-}} u^{\delta_{3}} d \mathcal{M}\right)^{1 / \delta_{3}} \leq C_{4} \inf _{Q^{+}} u
$$

where $C_{4}$ and $\delta_{3}$ depend only on $n$ and the structure conditions.

Remark 4.1. The conclusions of Theorem 4.1 and Corollary 4.1 are still valid, if $Q^{-}$ is replaced by $\widetilde{Q}\left(\bar{z}, \frac{3}{2} R\right)$. To this end, in the proof of Theorem 4.1, we just need to take $M$ and $\bar{K}$ so large that $\frac{3}{2} \leq \tau_{i} \leq 2$.

Next we will estimate $\sup u$ by the weak Harnack inequality. For $z_{0}=\left(x_{0}, t_{0}\right)$, let $Q_{0}=Q\left(z_{0}, \frac{3}{2} R\right)$, and a smaller section $Q_{s}=S\left(x_{0}, \frac{5}{4} R\right) \times\left(t_{0}-\frac{11}{8} R, t_{0}\right]$.

Lemma 4.2. Let $u \geq 0$ satisfy (1-1) in $Q_{0}$. Suppose that

$$
\iint_{Q_{0}} u^{\delta_{3}} d \mathcal{M} \leq C_{5} \mathcal{M}\left(Q_{0}\right)
$$


Let $\nu=\frac{M_{0}}{M_{0}-\frac{1}{2}}$ where $M_{0}$ is the constant in Lemma 3.1. Then there exist $C_{6}$, $\delta_{4}$, and $j_{0}$ depending only on $n, C_{5}, \delta_{3}$ and the structure conditions such that for $z^{\prime}=\left(x^{\prime}, t^{\prime}\right) \in Q_{s}$ and $j \geq j_{0}$, if $u\left(z^{\prime}\right)>\nu^{j-1} M_{0}$, then

$$
\sup _{Q\left(z^{\prime}, \rho\right)} u>\nu^{j} M_{0}
$$

where $\rho=C_{6} \nu^{-\delta_{4} j} R$ and $Q\left(z^{\prime}, \rho\right) \subset Q_{0}$.

Proof. By the properties of sections, if $j_{0}$ is sufficiently large, then $Q\left(z^{\prime}, \rho\right) \subset Q_{0}$. Suppose that $\sup _{Q\left(z^{\prime}, \rho\right)} u \leq \nu^{j} M_{0}$. Consider

$$
w(z)=\frac{\nu^{j} M_{0}-u(z)}{\nu^{j-1}(\nu-1) M_{0}} .
$$

Obviously, $w \geq 0$ and $w$ satisfies $(1-1)$ in $Q\left(z^{\prime}, \rho\right)$. It is easy to check that $w\left(z^{\prime}\right) \leq 1$.

Applying Lemma 3.1 to $w$, we obtain

$$
\mathcal{M}\left(\left\{z \in Q\left(z^{\prime}, \rho\right): w(z)<M_{0}\right\}\right)>\varepsilon_{0} \mathcal{M}\left(Q\left(z^{\prime}, \rho\right)\right),
$$

which is equivalent to the following:

$$
\mathcal{M}\left(\left\{z \in Q\left(z^{\prime}, \rho\right): u(z)>\frac{1}{2} \nu^{j} M_{0}\right\}\right)>\varepsilon_{0} \rho \mu\left(S\left(x^{\prime}, \rho\right)\right) .
$$

By (4-15) we have

$$
\left(\frac{1}{2} \nu^{j} M_{0}\right)^{-\delta_{3}} C_{5} \frac{3}{2} R \mu\left(S\left(x_{0}, \frac{3}{2} R\right)\right)>\varepsilon_{0} \rho \mu\left(S\left(x^{\prime}, \rho\right)\right) .
$$

Since $\mu$ is a doubling measure, we have

$$
\begin{aligned}
\mu\left(S\left(x_{0}, \frac{3}{2} R\right)\right) & \leq \mu\left(S\left(x^{\prime}, \theta \frac{3}{2} R\right)\right) \\
& \leq C\left(\frac{R}{\rho}\right)^{d} \mu\left(S\left(x^{\prime}, \rho\right)\right) .
\end{aligned}
$$

By (4-16) we obtain

$$
C \nu^{-\delta_{3} j} R^{1+d}>\rho^{1+d}
$$

which gives a contradiction if $\rho=\left(C \nu^{-\delta_{3} j}\right)^{1 / 1+d} R$. Therefore, we complete the proof of the lemma.

Theorem 4.2. Let $u \geq 0$ satisfy (1-1) in $Q_{0}$. Suppose that (4-15) holds. Then there exists $j_{1}$ such that $\sup _{\widehat{Q}} u \leq \nu^{j_{1}-1} M_{0}$, where $\widehat{Q}=S\left(x_{0}, R\right) \times\left(t_{0}-\frac{5 R}{4}, t_{0}\right]$ and $j_{1}$ depends only on $n, C_{5}, \delta_{3}, \delta_{4}$ and the structure conditions.

Proof. Choose $j_{1} \geq j_{0}$ such that

$$
\sum_{j \geq j_{1}} C_{6} \nu^{-\delta_{4} j}<\frac{1}{12}
$$

and

$$
\sum_{j \geq j_{1}}\left(C_{6} \nu^{-\delta_{4} j}\right)^{1 / p}<\frac{1}{6}
$$

where $p$ is the constant in Property (D) in $\S 2$. We claim that $\sup _{\widehat{Q}} u \leq \nu^{j_{1}-1} M_{0}$. Otherwise, suppose that there exists $z^{\prime} \in \widehat{Q}$ such that $u\left(z^{\prime}\right)>\nu^{j_{1}-1} M_{0}$. By Lemma 
4.2, there exists $z_{j_{1}} \in Q\left(z^{\prime}, \rho_{j_{1}}\right)$ with $\rho_{j_{1}}=C_{6} \nu^{-\delta_{4} j_{1}} R$ such that $u\left(z_{j_{1}}\right)>\nu^{j_{1}} M_{0}$. By (4-17) and (4-18), $z_{j_{1}} \in Q_{s}$. Apply Lemma 4.2 again and obtain $z_{j_{1}+1} \in$ $Q\left(z_{j_{1}}, \rho_{j_{1}+1}\right)$ with $\rho_{j_{1}+1}=C_{6} \nu^{-\delta_{4}\left(j_{1}+1\right)} R$ such that $u\left(z_{j_{1}+1}\right)>\nu^{j_{1}+1} M_{0}$. By (4-17) and (4-18), $z_{j_{1}+1} \in Q_{s}$. Continue this process and we obtain that there exists $\left\{z_{k}\right\}_{j_{1}}^{\infty} \subset Q_{s}$ such that $u\left(z_{k}\right)>\nu^{k} M_{0}$ which contradicts the continuity of $u$. Hence, we finish the proof.

Proof of Theorem 1.1. Choose $\bar{\theta}=8 \theta^{2}$ in Theorem 1.1, where $\theta$ is the engulfing constant. Then Theorem 1.1 is an immediate consequence of the combination of Corollary 4.1 and Theorem 4.2.

Remark 4.2. By Theorem 1.1 and a standard argument, we conclude

$$
\operatorname{osc}_{Q\left(z_{0}, \rho\right)} u \leq C^{\prime}\left(\frac{\rho}{R}\right)^{\alpha} \operatorname{osc}_{Q\left(z_{0}, R\right)} u, \quad \text { for } \rho<R,
$$

where $C^{\prime}$ and $\alpha$ are constants. For the proof, see [M].

Remark 4.3. We show that Remark 4.2 implies Hölder continuity. But the Hölder constant depends on the norm of the affine transformation which normalizes the section under consideration. Let $Q\left(z_{0}, R\right)$ be a section and $(x, t),(y, s) \in Q\left(z_{0}, R / 2\right)$ with $s \leq t$. Let

$$
\rho_{0}=\inf \{\rho: y \in \overline{S(x, \rho)}\} .
$$

By [Ca-Gu2], we have

$$
\rho_{0} \leq 2 \theta R\left(\frac{2\|T\|}{K_{2}}\right)^{1 / \varepsilon_{3}}|x-y|^{1 / \varepsilon_{3}},
$$

where $\|T\|$ denotes the norm of $T$ which normalizes $S\left(x_{0}, \theta R\right)$. Therefore

$$
(y, s) \in Q\left((x, t), C|x-y|^{1 / \varepsilon_{3}}+|t-s|\right) .
$$

By Remark 4.2, it follows that

$$
|u(x, t)-u(y, s)| \leq C\left(|x-y|^{1 / \varepsilon_{3}}+|t-s|\right)^{\alpha} R^{-\alpha},
$$

where $C$ depends on $\|T\|,\|u\|_{L^{\infty}}, n$, and constants in structure conditions.

\section{ACKNOWLEDGEMENT}

This is a part of my Ph.D. dissertation. The author is very indebted to his dissertation advisor, Professor Cristian E. Gutiérrez, for leading him to this problem, his patient guidance, and constant encouragement. The author also expresses his gratitude to the referee for some suggestions.

\section{REFERENCES}

[Ca1] L.A. Caffarelli, Some regularity properties of solutions of Monge-Ampère equation, Comm. Pure Appl. Math. XLIV (1991), 965-969. MR 92h:35088

[Ca2] Boundary regularity of maps with convex potentials, Comm. Pure Appl. Math. XLV (1992), 1141-1151. MR 93k:35054

[Ca-C] L. A. Caffarelli and X. Cabré, Fully nonlinear elliptic equations, AMS Colloquium Publications V. 43, AMS, Rhode Island, 1993. MR 96h:35046

[Ca-Gu1] L. A. Caffarelli and C. E. Gutiérrez, Real analysis related to the Monge-Ampère equation, Trans. Amer. Math. Soc. 348 (1996), 1075-1092. MR 96h:35047

[Ca-Gu2] _ Properties of the solutions of the linearized Monge-Ampère equation, Amer. J. of Math. 119 (1997), 423-465. MR 98e:35060 
[G-T] D. Gilbarg \& N. S. Trudinger, Elliptic partial differential equations of second order, 2nd edition, Springer-Verlag, 1983. MR 86c:35035

[Gu] C. E. Gutiérrez, On the Harnack inequality for viscosity solutions of non-divergence equations, preprint.

[Gu-H] C. E. Gutiérrez \& Q. Huang, Geometric properties of the sections of solutions of the Monge-Ampère equation, Trans. AMS. to appear.

[K-S] N. V. Krylov \& M. V. Safonov, Certain properties of solutions of parabolic equations with measurable coefficients, Izvestia Akad. Nauk. SSSR 44 (1980), 161-175. MR 83c:35059

[M] J. Moser, A Harnack inequality for parabolic differential equations, Comm. Pure. Appl. Math. 17 (1964), 101-134. MR 28:2356

[S] E. M. Stein, Harmonic analysis: real-variable methods, orthogonality, and oscillatory integrals, Princeton Math. Series \#43, Princeton U. Press, Princeton, NJ, 1993. MR 95c: 42002

[T] K. S. Tso, On an Alekandrov-Bakel'man type maximum principle for second-order parabolic equations, Comm. PDE 10 (1985), 543-553. MR 87f:35031

[W] L. Wang, On the regularity theory of fully nonlinear parabolic equations I, Comm. Pure Appl. Math. 45 (1992), 27-76. MR 92m:35126

[W-W] Rou-Huai Wang \& Guang-Lie Wang, On existence, uniqueness and regularity of viscosity solutions for the first initial-boundary value problems to parabolic Monge-Ampère equation, Northeast. Math. J. 8 (1992), 417-446. MR 94d:35085

Department of Mathematics, Temple University, Philadelphia, Pennsylvania 19122

Current address: Department of Mathematics, University of Texas at Austin, Austin, Texas 78712

E-mail address: qhuang@math.utexas.edu 